\title{
New gravitational solutions via a Riemann-Hilbert approach
}

\author{
G.L. Cardoso ${ }^{a}$ and J.C. Serra ${ }^{b}$ \\ ${ }^{a}$ Center for Mathematical Analysis, Geometry and Dynamical Systems, \\ Department of Mathematics, Instituto Superior Técnico, Universidade de Lisboa, \\ Av. Rovisco Pais, 1049-001 Lisboa, Portugal \\ ${ }^{b}$ Instituto Superior Técnico, Universidade de Lisboa, \\ Av. Rovisco Pais, 1049-001 Lisboa, Portugal \\ E-mail: gcardoso@math.tecnico.ulisboa.pt, \\ joao. leandro.camara.serra@tecnico.ulisboa.pt
}

ABSTRACT: We consider the Riemann-Hilbert factorization approach to solving the field equations of dimensionally reduced gravity theories. First we prove that functions belonging to a certain class possess a canonical factorization due to properties of the underlying spectral curve. Then we use this result, together with appropriate matricial decompositions, to study the canonical factorization of non-meromorphic monodromy matrices that describe deformations of seed monodromy matrices associated with known solutions. This results in new solutions, with unusual features, to the field equations.

KeYwords: 2D Gravity, Black Holes, Integrable Field Theories, Sigma Models

ARXIV EPRINT: 1711.01113 


\section{Contents}

1 Introduction 1

2 The Breitenlohner-Maison linear system and canonical factorization 4

3 New solutions by deformation of seed monodromy matrices $\quad 8$

3.1 Deformed monodromy matrices in dimensionally reduced Einstein-Maxwelldilaton theory 8

3.2 Deformed monodromy matrices in dimensionally reduced Einstein gravity 18 3.2.1 Deforming the monodromy matrix of the Schwarzschild solution 21

A Explicit factorization

\section{Introduction}

The Riemann-Hilbert factorization approach to solving the field equations of gravity theories is remarkable in that it allows to study the subspace of solutions to the field equations that only depend on two space-time coordinates, in terms of the canonical factorization of a so-called monodromy matrix into matrix factors $M_{ \pm}$with prescribed analiticity properties in a complex variable $\tau$ [1]. Thus, instead of directly solving non-linear PDE's in two variables, the problem of solving the field equations is mapped to a canonical Riemann-Hilbert factorization problem in one complex variable $\tau$. Let us describe this approach.

We consider the dimensional reduction of gravity theories (without a cosmological constant) to two spatial dimensions. The resulting two-dimensional effective action describes a scalar non-linear sigma model coupled to gravity. The equations of motion for the scalar fields can be recast in terms of an auxiliary linear system, called the Breitenlohner-Maison linear system [1], which depends on an additional parameter, the spectral parameter $\tau \in \mathbb{C}$. The solvability condition for the linear system yields the equations of motion for the scalar fields [1-3], provided $\tau$ is taken to be a position dependent spectral parameter that satisfies the relation

$$
\omega=v+\frac{1}{2} \rho \frac{\left(1-\tau^{2}\right)}{\tau},
$$

which defines an algebraic curve. Here $\omega \in \mathbb{C}$, while $(\rho, v) \in \mathbb{R}^{2}$ denote two space-like coordinates, called Weyl coordinates. Locally, the relation (1.1) can be inverted to yield $\tau=\tau(\omega, \rho, v)$, so that $\tau$ is a function of the Weyl coordinates that parametrize the twodimensional space and of a complex parameter $\omega$. While here we consider the case when $(\rho, v)$ are space-like coordinates, the case of one time-like and one space-like coordinate can be treated in a similar manner $[2,3]$. 
Given a solution to the linear system, one can associate to it a so-called monodromy matrix $\mathcal{M}(\omega)$, whose entries only depend on the parameter $\omega$. Conversely, given a candidate monodromy matrix $\mathcal{M}(\omega)$, one may ask if, for $\omega$ given by (1.1), it possesses a so-called canonical factorization, as follows. One considers a closed single contour $\Gamma$ in the complex $\tau$-plane, which we take to be the unit circle centered around the origin, i.e. $\Gamma=\{\tau \in$ $\mathbb{C}:|\tau|=1\}$. This contour divides the complex plane $\mathbb{C}$ into two regions, namely the interior and the exterior region of the unit circle $\Gamma$. Then, given a matrix $\mathcal{M}(\omega(\tau))$, with an inverse $\mathcal{M}^{-1}(\omega(\tau))$, such that both are continuous on $\Gamma$, one seeks a decomposition $\mathcal{M}(\omega(\tau))=M_{-}(\tau) M_{+}(\tau)$, valid on $\Gamma$, where $M_{ \pm}$have to satisfy certain analiticity and boundedness conditions, to be reviewed in section 2 . In particular, $M_{-}$is analytic in the exterior region, while $M_{+}$is analytic in the interior region.

If $\mathcal{M}(\omega)$ possesses a canonical factorization, then the latter is unique, up to multiplication by a constant matrix. This freedom can be fixed by imposing a normalization condition on one of the factors, say on $M_{+}$. Then, it can be shown [4] that the factor $M_{-}$ yields a solution to the equations of motion for the scalar fields in two dimensions, and consequently of the gravitational field equations. The factor $M_{+}$, on the other hand, yields a solution to the linear system.

The question of the existence of a canonical factorization for $\mathcal{M}(\omega)$ is an example of a matricial Riemann-Hilbert factorization problem. In the Riemann-Hilbert approach to gravity, rather than solving the non-linear field equations directly, one is instructed to perform the canonical factorization of a monodromy matrix $\mathcal{M}(\omega)$ in order to obtain a solution to the field equations. To be able to obtain explicit solutions to the field equations, the canonical factorization must be performed explicitly. While this is, in general, well understood for scalar functions [5, 6], the situation changes dramatically in the case of matrix functions. For the latter, it is not known in general whether such a factorization exists; and in case it exists, there are no general methods available for obtaining it explicitly. Thus, different methods have to be developed on a case by case basis for different classes of matrices. The monodromy matrices considered recently in the literature have either simple poles [7-12] or double and simple poles [4] in the $\omega$-plane. In [4] the corresponding matricial Riemann-Hilbert factorization problem was converted into a vectorial RiemannHilbert factorization problem, which was subsequently solved using a generalization of Liouville's theorem. The explicit factorization method that was applied to monodromy matrices with single or double poles in [4] is of greater generality, but it can be applied only to rational monodromy matrices; the factorization of non-rational monodromy matrices requires developing other factorization methods.

Which monodromy matrices should one then pick as a starting point? One strategy for choosing $\mathcal{M}(\omega)$ consists in starting from the monodromy matrix associated to a known solution to the field equations, and then deforming this monodromy matrix to obtain a different matrix to be factorized. This is the strategy that we will adopt in this paper. In doing so, we first study monodromy matrices of the form $\mathcal{M}(\omega)=f(\omega) \tilde{\mathcal{M}}(\omega)$, where $f(\omega)$ is a scalar function. Here we obtain our first result, as follows. Take the unit circle contour $\Gamma$ in the $\tau$-plane, and consider its image in the $\omega$-plane by means of the algebraic curve relation (1.1). We will denote the resulting closed contour in the $\omega$-plane by $\Gamma_{\omega}$. As 
we will show in section 2 , any rational function $f$ in $\omega$ that has no zeroes and no poles on $\Gamma$ has a canonical factorization in $\tau \in \Gamma$. Moreover, any function $f(\omega)$ that is continuous and non-vanishing on $\Gamma_{\omega}$ also has a canonical factorization. This surprising result is quite unexpected in view of the existing results on the factorization of scalar functions $[5,6]$ (which is not, in general, canonical), and turns out to be a consequence of the StoneWeierstrass theorem when combined with properties of the algebraic curve relation (1.1).

Armed with this result, we turn to the study of solutions of two four-dimensional gravity theories in section 3, namely Einstein gravity and Einstein-Maxwell-dilaton theory. The latter is obtained by performing a Kaluza-Klein reduction of five-dimensional Einstein gravity. We begin with the study of solutions of Einstein-Maxwell-dilaton theory. As a starting point we pick the solution that describes an $A d S_{2} \times S^{2}$ space-time, supported by electric/magnetic charges $(Q, P)$ and a constant dilaton scalar field. The associated monodromy matrix was given in [4], and its entries contain double and simples poles at $\omega=0$. We then perform a two-parameter deformation of this monodromy matrix. The resulting monodromy matrix is of the form $\mathcal{M}(\omega)=f(\omega) \tilde{\mathcal{M}}(\omega)$, where $\tilde{\mathcal{M}}(\omega)$ is a matrix with entries that are rational functions with double or simple poles, while $f(\omega)$ is given by the third root of a rational function $g(\omega)$. Since $g(\omega)$ possesses a canonical factorization in view of the theorem mentioned above, also $f(\omega)$ will possess a canonical factorization, provided we pick the branch cuts of the roots appropriately. Then, using the explicit factorization method presented in [4] and alluded to above, we perform the explicit canonical factorization of $\tilde{\mathcal{M}}(\omega)$. The resulting space-time solution has unusual features. It describes a stationary solution that is supported by a non-constant oscillatory dilaton field. It interpolates between an asymptotic space-time with a NUT parameter $J$ and a Killing horizon. The near-horizon geometry is not $A d S_{2} \times S^{2}$, but nevertheless has a deep throat, and as a result the dilaton field exhibits the behaviour of a static attractor $[13,14]$ : due to the deep throat in the geometry, the dilaton field flows to a constant value that is entirely specified by the electric/magnetic charges $(Q, P)$. This unusual stationary solution is complicated: it is given in terms of a power series in $J$, and it results from the particular deformation of the monodromy matrix that we chose. To obtain this solution by directly solving the field equations is likely not to be straightforward.

Next, we turn to four-dimensional Einstein gravity and study deformations of the monodromy matrix associated to the Schwarzschild solution. The monodromy matrices that arise in four-dimensional Einstein gravity are $2 \times 2$ symmetric matrices with entries determined in terms of three functions, which we denote by $a, b$ and $R^{2}$, see (3.46). These functions are continuous on $\Gamma_{\omega}$, with $b^{2} R^{2}-a^{2}=1$ on $\Gamma_{\omega}$. We show that any such matrix can be decomposed as in (3.47) into matrix factors whose entries only depend on the combinations $a \pm b R$ and on $R$. To proceed, we have to make a choice for the functions $R$. We take $R$ to be a rational function of $\omega$, with no zeroes and no poles on $\Gamma_{\omega}$, and similarly for its inverse $R^{-1}$. Note that the Schwarzschild solution is captured by this class of functions, and this motivates the choice of this class. With this choice, the combinations $a \pm b R$ possess a canonical factorization in view of the theorem that we prove in section 2 and that we mentioned above. For this choice of functions $R$, we obtain a class of monodromy matrices whose canonical factorization can be performed regardless of the type of isolated singular- 
ities that the combinations $a \pm b R$ may have. We illustrate this by choosing combinations $a \pm b R$ that have an essential singularity at $\omega=0$. The resulting monodromy matrix describes a deformation of the monodromy matrix associated with the Schwarzschild solution. We show that the presence of this essential singularity does not pose any problem for explicitly performing the canonical factorization of the deformed matrix. This exemplifies that it is possible to perform canonical factorizations of monodromy matrices that have more complicated singularities than the rational ones considered in the recent literature.

Some of the conclusions that we draw from these explicit factorizations are as follows. First, factorization transforms innocently looking deformations of monodromy matrices into highly non-trivial deformations of space-time solutions. Second, one may ask whether continuity in the deformation parameters is preserved by the factorization. We have analysed this question for one of the deformation parameters in the example that we studied in the context of four-dimensional Einstein-Maxwell-dilaton theory, and we find that this is indeed the case. Third, the deformed space-time solutions that result by using the Riemann-Hilbert factorization approach may be difficult to obtain by direct means, i.e. by directly solving the four-dimensional field equations. This constitutes one of the advantages of the factorization approach. And finally, due to the aforementioned theorem that we prove in section 2 and to an appropriate decomposition of the monodromy matrix, we find that the difficulties in factorizing monodromy matrices with singularities that are not just poles can be overcome. This in turn raises a question, which we will not address here: is there a correspondence between the type of singularities in the $\omega$-plane and properties of the associated space-time solution?

\section{The Breitenlohner-Maison linear system and canonical factorization}

We consider the dimensional reduction of four-dimensional gravity theories (at the twoderivative level, and without a cosmological constant) down to two dimensions [1]. The resulting theory in two dimensions can be brought to the form of a scalar non-linear sigmamodel coupled to gravity. We take the sigma-model target space to be a symmetric space $G / H$. In performing the reduction, we first reduce to three dimensions over a time-like isometry direction. We denote the associated three-dimensional line element by

$$
d s_{3}^{2}=e^{\psi}\left(d \rho^{2}+d v^{2}\right)+\rho^{2} d \phi^{2} .
$$

Here, $\psi$ is a function of the coordinates $(\rho, v)$, which are called Weyl coordinates. Throughout this paper, we take $\rho>0$. Subsequently, we reduce to two dimensions along the space-like isometry direction $\phi$.

The resulting equations of motion in two dimensions take the form [1]

$$
d(\rho \star A)=0,
$$

where $d$ denotes the exterior derivative, and the matrix one-form $A=A_{\rho} d \rho+A_{v} d v$ equals

$$
A=M^{-1} d M .
$$


Here, $M(\rho, v)$ denotes the representative of the symmetric space $G / H$, and it satisfies $M=M^{\natural}$, where the operation $\natural$ denotes a 'generalized transposition' that acts antihomomorphically on matrices [3]. The operation $\star$ denotes the Hodge dual in two dimensions. The warp factor $\psi$ in (2.1) is then obtained by integrating $[2,3]$

$$
\begin{aligned}
\partial_{\rho} \psi & =\frac{1}{4} \rho \operatorname{Tr}\left(A_{\rho}^{2}-A_{v}^{2}\right), \\
\partial_{v} \psi & =\frac{1}{2} \rho \operatorname{Tr}\left(A_{\rho} A_{v}\right) .
\end{aligned}
$$

The equations of motion (2.2) for $M$ can be reformulated in terms of an auxiliary linear system, the so-called Breitenlohner-Maison linear system, ${ }^{1}$ whose solvability implies (2.2). This linear system reads $[2,3]$

$$
\tau(d+A) X=\star d X .
$$

It depends on a complex parameter $\tau$, called the spectral parameter. To ensure that the solvability of (2.5) implies the equations of motion (2.2), $\tau$ has to satisfy the algebraic curve relation

$$
\omega=v+\frac{\rho}{2 \tau}\left(1-\tau^{2}\right)
$$

where $\omega \in \mathbb{C}$. With $\rho>0$, this results in

$$
\tau(\omega, \rho, v)=\frac{1}{\rho}\left(v-\omega \pm \sqrt{\rho^{2}+(v-\omega)^{2}}\right), \quad \omega \in \mathbb{C} .
$$

Let us assume that there exists a pair $(A, X)$ (with $\left.A=M^{-1} d M\right)$ that solves $(2.5)$ such that $X$ and its inverse $X^{-1}$ are analytic in $\tau$, for $\tau$ in the interior of the unit circle $\Gamma$ (centered around the origin in the $\tau$-plane), with continuous boundary valued funtions on $\Gamma$. Then, $A=M^{-1} d M$ is a solution to the equations of motion (2.2), and one can assign to the pair $(M, X)$ a so-called monodromy matrix $\mathcal{M}(\omega)$ that satisfies $\mathcal{M}(\omega)=\mathcal{M}^{\natural}(\omega)$ and possesses a canonical factorization,

$$
\mathcal{M}(\omega(\tau, \rho, v))=M_{-}(\tau, \rho, v) M_{+}(\tau, \rho, v), \quad \tau \in \Gamma .
$$

Here, on the left hand side, $\omega$ is viewed as a function of $\tau \in \mathbb{C}$ using the relation (2.6), and both $\mathcal{M}$ and its inverse are continuous on $\Gamma$. The factorization (2.8) is valid in the $\tau$-plane, with respect to the unit circle $\Gamma$ centered around $\tau=0$. The factors $M_{ \pm}$are such that: $M_{+}$ and its inverse $M_{+}^{-1}$ are analytic and bounded in the interior of the unit circle $\Gamma$, while $M_{-}$ and its inverse $M_{-}^{-1}$ are analytic and bounded in the exterior of the unit circle $\Gamma$. Also, $M_{+}$is normalised to $M_{+}(\tau=0)=\mathbb{I}$, rendering the factorization unique. We refer to [4] for a comprehensive review of the conditions for the existence of a canonical factorization.

Conversely, consider a monodromy matrix $\mathcal{M}(\omega)$ that satisfies $\mathcal{M}=\mathcal{M}^{\natural}$ and possesses a canonical factorization (2.8) (with $\omega$ given as in (2.6)) satisfying $M_{+}(\tau=0)=\mathbb{I}$. Then, it can be shown that $M(\rho, v)=M_{-}(\tau=\infty, \rho, v)$ is a solution to the equations of motion (2.2),

\footnotetext{
${ }^{1}$ In this paper, we work with the Breitenlohner-Maison linear system. There exists another linear system, the Belinski-Zakharov linear system, that is often used to address the construction of solitonic solutions. We refer to [15] and references therein for a discussion of the relation between these two linear systems.
} 
and $M_{+}(\tau, \rho, v)$ is a solution to the linear system (2.5) [4]. Thus, in this approach, to obtain explicit solutions to the field equations, we first pick a monodromy matrix $\mathcal{M}(\omega)$ that possesses a canonical factorization, then perform the factorization explicitly to extract the factors $M_{ \pm}$, to obtain $M(\rho, v)=M_{-}(\tau=\infty, \rho, v)$, which encodes the space-time solution. Note that the condition (2.8), or equivalently the jump condition $\mathcal{M} M_{+}^{-1}=M_{-}$on $\Gamma$, defines a matrix Riemann-Hilbert problem.

Necessary and sufficient conditions for the existence of a canonical factorization $\mathcal{M}=$ $M_{-} M_{+}$were summarized in [4], where a method for obtaining explicit factorizations was also described. This method, based on solving a vectorial Riemann-Hilbert problem $\mathcal{M} \phi_{+}=\phi_{-}$by means of Liouville's theorem (or a generalization thereof), is the one that we will follow throughout. Here, the $\phi_{+}$denote the columns of $M_{+}^{-1}$, while the $\phi_{-}$ denote the columns of $M_{-}$.

The monodromy matrices that we will factorize will be of the type $\mathcal{M}(\omega)=f(\omega) \tilde{\mathcal{M}}(\omega)$, where $f(\omega)$ denotes a function, and $\tilde{\mathcal{M}}$ a matrix. Since we are interested in monodromy matrices that have a canonical factorization, i.e. a factorization of the form (2.8) with $M_{ \pm}$ satisfying the analyticity and boundedness properties described in the text below (2.8), which class of functions $f(\omega)$ should we consider to ensure that $f(\omega)$ has a canonical factorization (i.e. $f(\omega)=f_{+}(\tau) f_{-}(\tau)$ with $f_{ \pm}$satisfying the analyticity and boundedness properties described in the text below (2.8))? Here we obtain the following surprising result:

Theorem. Any function $f(\omega)$ that is continuous and non-vanishing on $\Gamma_{\omega}$ has a canonical factorization. Here, $\Gamma_{\omega}$ denotes the image of the curve $\Gamma$ (the unit circle in the $\tau$-plane centered at $\tau=0$ ) under (2.6).

Proof. The proof uses the Stone-Weierstrass theorem and the algebraic curve relation (2.6).

The Stone-Weierstrass theorem states that if $K$ is a compact subset of $\mathbb{C}$, then every continuous, complex-valued function $f$ on $K$ can be uniformly approximated by polynomials $P_{n}$ in $\omega$ and $\bar{\omega}$, i.e.

$$
\sup _{\omega \in K}\left|f(\omega)-P_{n}(\omega, \bar{\omega})\right| \rightarrow 0, \quad n \rightarrow \infty, \quad n \in \mathbb{N} .
$$

Here we take $K=\Gamma_{\omega}$, where $\Gamma_{\omega}$ denotes the image of the curve $\Gamma$ under (2.6). Then, using (2.6), we obtain the relation $\bar{\omega}=-\omega+2 v$, which holds for any $\omega \in \Gamma_{\omega}$. Thus, viewed as functions of $\omega \in \Gamma_{\omega}$, the $\tilde{P}_{n}(\omega)=P_{n}(\omega, \bar{\omega})$ are polynomials in $\omega$, while viewed as functions of $\tau \in \Gamma$, the $\tilde{P}_{n}(\omega(\tau))$ are rational functions in $\tau$. We will show momentarily that any rational function in $\omega$, when seen as a function of $\tau \in \Gamma$ via composition with $\omega(\tau)$, has a canonical factorization in $\tau \in \Gamma$, so long as it has no zeroes and no poles on $\Gamma$. Then, since $\sup _{\tau \in \Gamma}\left|f(\omega(\tau))-\tilde{P}_{n}(\omega(\tau))\right|=\sup _{\omega \in K}\left|f(\omega)-\tilde{P}_{n}(\omega)\right|, f(\omega(\tau))$ is uniformly approximated by rational functions on $\Gamma$ that possess a canonical factorization. Therefore, $f$ also has a canonical factorization (see, for instance, section 5 of the review paper [6] and references therein).

The same conclusion is reached by looking at the index of the function $f(\omega(\tau))$. This index is defined as follows [5]. We consider a function $f(\omega)$ that is continuous and nonvanishing on $\Gamma_{\omega}$, and hence also continuous and non-vanishing on $\Gamma$. Let us represent the image of the function $f(\omega(\tau))$ in a complex plane, which we will call the $F$-plane. We 
denote the image of $\Gamma$ under $f(\omega(\tau))$ by $\Gamma_{f}$. Then, $\Gamma_{f}$ is a closed contour in the $F$-plane that does not pass through the origin of the $F$-plane. The index of $f$ is then defined to be the winding number of the closed contour $\Gamma_{f}$ around the origin of the $F$-plane. If this winding number is zero, then $f(\omega(\tau))$ possesses a canonical factorization by a known theorem [5], which states that any continuous function on $\Gamma$ with zero index admits a canonical factorization. We proceed to show that the winding number is zero.

The relation (2.6) associates two values of $\tau$, given by (2.7), to any $\omega$. Denoting these two values by $\tau_{1}$ and $\tau_{2}$, we have $\tau_{1} \tau_{2}=-1$. This means that there are two values of $\tau \in \Gamma$ that correspond to the same $\omega \in \Gamma_{\omega}$, namely $\tau_{1} \in \Gamma$ and $-\bar{\tau}_{1} \in \Gamma$. Now consider going around $\Gamma$ once, counterclockwise, starting at $\tau=-i$. In doing so, let us denote the directed curve starting at $\tau=-i$ and ending at $\tau=i$ by $\Gamma_{1}$, while the directed curve starting at $\tau=i$ and ending at $\tau=-i$ will be denoted by $\Gamma_{2}$. If we denote the image of $\Gamma_{1}$ under (2.7) by $\gamma_{\omega} \subset \Gamma_{\omega}$, then the image of $\Gamma_{2}$ under (2.7) is $-\gamma_{\omega}$. Therefore, if we go around $\Gamma$ once in a counterclockwise fashion, the closed curve $\Gamma_{\omega}$ that is travelled in the $\omega$-plane is $\gamma_{\omega}-\gamma_{\omega}$. Hence, if $f(\omega)$ is continuous and non-vanishing on $\Gamma_{\omega}$, the resulting contour $\Gamma_{f}$ has zero winding number with respect to the origin of the $F$-plane, and we conclude that $f(\omega(\tau))$ has a canonical factorization.

Finally, let us show that any rational function in $\omega$ has a canonical factorization in $\tau \in \Gamma$, so long as it has no zeroes and no poles on $\Gamma$. We begin by writing (2.6) in the form

$$
\omega-\omega_{0}=-\frac{\rho\left(\tau-\tau_{0}^{+}\right)\left(\tau-\tau_{0}^{-}\right)}{2 \tau}
$$

where $\tau_{0}^{+}, \tau_{0}^{-}$are the two values of $\tau$ corresponding to $\omega=\omega_{0}$. We assume that $\tau_{0}^{ \pm}$do not lie on the unit circle $\Gamma$ in the $\tau$-plane, and we take $\tau_{0}^{+}$to lie inside and $\tau_{0}^{-}$to lie outside the unit disc. Now consider a rational function $f(\omega)$ with $A_{1}$ zeroes and $A_{2}$ poles (counting multiplicities). When viewed as a function of $\tau$ using $(2.6), f(\omega(\tau))$ takes the form

$$
f(\omega(\tau))=\left(-\frac{\rho}{2 \tau}\right)^{A_{1}-A_{2}} \frac{\prod_{i=1}^{A_{1}}\left(\tau-\tau_{i}^{+}\right)}{\prod_{j=1}^{A_{2}}\left(\tau-\tau_{j}^{+}\right)} \frac{\prod_{i=1}^{A_{1}}\left(\tau-\tau_{i}^{-}\right)}{\prod_{j=1}^{A_{2}}\left(\tau-\tau_{j}^{-}\right)},
$$

up to an overall normalization constant. Here we assume that none of the zeroes and poles of $f(\omega(\tau))$ lie on the unit circle $\Gamma$, so that $f(\omega(\tau))$ and its inverse are continuous on $\Gamma$. Then, $\tau_{i}^{+}$and $\tau_{i}^{-}$are the two values of $\tau$ corresponding to the zero $\omega=\omega_{i}$, with $\left|\tau_{i}^{+}\right|<1$ and $\left|\tau_{i}^{-}\right|>1$, and similarly for the two values $\tau_{j}^{+}$and $\tau_{j}^{-}$associated with the pole $\omega=\omega_{j}$. The function $f(\omega(\tau))$ possesses a canonical factorization, $f=f_{-} f_{+}$, if $f_{-}$and its inverse are analytic in the exterior of the unit disc and bounded at $\tau=\infty$, and if $f_{+}$and its inverse are analytic and bounded in the interior of the unit disc . This is indeed the case, as can be verified by taking

$$
f_{-}=\left(-\frac{\rho}{2 \tau}\right)^{A_{1}-A_{2}} \frac{\prod_{i=1}^{A_{1}}\left(\tau-\tau_{i}^{+}\right)}{\prod_{j=1}^{A_{2}}\left(\tau-\tau_{j}^{+}\right)}, \quad f_{+}=\frac{\prod_{i=1}^{A_{1}}\left(\tau-\tau_{i}^{-}\right)}{\prod_{j=1}^{A_{2}}\left(\tau-\tau_{j}^{-}\right)}
$$


Note that, while it is true that any rational function in $\tau$ has a factorization, the latter is in general not canonical (see, for instance, [6] and references therein). However, in our case, due to the spectral curve relation between $\omega$ and $\tau$, any rational function in $\omega$ has a canonical factorization in $\tau$, provided that it has no zeroes nor poles on the unit circle $\Gamma$.

In particular, if a rational function $f(\omega(\tau))$ has a canonical factorization, $f=f_{-} f_{+}$, then its $n$-th root also has one. This is a consequence of the following textbook theorem [16]: if $g(z)$ is analytic and non-vanishing in a simply connected region $\Omega$, then it is possible to define single-valued analytic branches of $\sqrt[n]{g(z)}$ in $\Omega$. Setting $g=f_{+}$, we obtain part of the assertion. For the factor $f_{-}$, we use the Schwarz reflection principle and consider $\tilde{f}_{+}=\bar{f}_{-}$, defined in the interior region of $\Gamma$ by $\tilde{f}_{+}(z)=\overline{f_{-}(1 / \bar{z})}$ and on $\Gamma$ by $\tilde{f}_{+}(z)=\overline{f_{-}(z)}$, which is analytic (extended to $z=0$ ) and non-vanishing in the unit disk. Hence, we can apply the theorem mentioned above to $\tilde{f}_{+}$to define an analytic branch of $\sqrt[n]{\tilde{f}_{+}(z)}$. Then, applying the Schwarz reflection principle to the latter, we obtain $\overline{\left(\sqrt[n]{\overline{f_{-}(z)}}\right)}$, which defines an analytic branch of $\sqrt[n]{f_{-}(z)}$, and the assertion follows.

Given a solution $M(\rho, v)$ of the two-dimensional equations of motion (2.2), it may be useful to have a rule that assigns a candidate monodromy matrix $\mathcal{M}(\omega)$ to it. One such rule is the so-called substitution rule given in [4], which consists in the following. Assuming that the limit $\lim _{\rho \rightarrow 0^{+}} M(\rho, v)$ exists, the candidate monodromy matrix is obtained by substituting $v$ by $\omega$ in this expression, i.e.

$$
\mathcal{M}(\omega=v)=\lim _{\rho \rightarrow 0^{+}} M(\rho, v) .
$$

Whether, upon performing a canonical factorization, this candidate monodromy matrix really yields back the solution $M(\rho, v)$ has to be verified case by case.

\section{New solutions by deformation of seed monodromy matrices}

In this section we construct new solutions to the dimensionally reduced gravitational field equations by deforming the monodromy matrices associated to known solutions. The monodromy matrices of the latter will be called seed monodromy matrices. The deformed monodromy matrices we consider fall into the class of monodromy matrices to which the theorem given in the previous section applies, and hence they possess a canonical factorization, which we carry out explicitly. We do this in the context of two gravitational theories, namely four-dimensional Einstein-Maxwell-dilaton theory (obtained by Kaluza-Klein reduction of five-dimensional Einstein gravity) and four-dimensional Einstein gravity theory.

We begin by considering the Einstein-Maxwell-dilaton theory.

\subsection{Deformed monodromy matrices in dimensionally reduced Einstein- Maxwell-dilaton theory}

The field equations of the four-dimensional Einstein-Maxwell-dilaton theory, that is obtained by Kaluza-Klein reducing five-dimensional Einstein gravity, admits extremal black hole solutions [17-22]. These solutions, which may be static or rotating, are supported by 
a scalar field $e^{-2 \Phi}$ (the dilaton field) and by an electric charge $Q$ and a magnetic charge $P$. We will take $Q>0, P>0$ throughout. An example of a static solution is the extremal Reissner-Nordström black hole solution, which is supported by a constant dilaton field $e^{-2 \Phi}=Q / P$, and which interpolates between flat space-time and an $A d S_{2} \times S^{2}$ space-time. In adapted coordinates, the four-dimensional line element of the latter reads

$$
d s_{4}^{2}=-\frac{r^{2}}{Q P} d t^{2}+Q P \frac{d r^{2}}{r^{2}}+Q P\left(d \theta^{2}+\sin ^{2} \theta d \phi^{2}\right) .
$$

In these coordinates, the extremal Reissner-Nordström solution interpolates between a flat space-time metric at $r=\infty$ and a near-horizon metric (3.1) at $r=0$.

The $A d S_{2} \times S^{2}$ space-time, described by (3.1) and supported by a constant dilaton field $e^{-2 \Phi}=Q / P$, is by itself a solution to the field equations of the Einstein-Maxwell-dilaton theory. As shown in [4], upon dimensional reduction to two dimensions, this solution can be associated with the following monodromy matrix,

$$
\mathcal{M}_{\text {seed }}(\omega)=\left(\begin{array}{ccc}
A / \omega^{2} & B / \omega & C \\
-B / \omega & D & 0 \\
C & 0 & 0
\end{array}\right), \quad \operatorname{det} \mathcal{M}=1
$$

where

$$
A=P^{4 / 3} Q^{2 / 3}, \quad B=\sqrt{2} P^{1 / 3} Q^{2 / 3}, \quad C=-\left(\frac{P}{Q}\right)^{1 / 3}, \quad D=-\left(\frac{Q}{P}\right)^{2 / 3} .
$$

Note that the entries in (3.2) are rational functions in the variable $\omega \in \mathbb{C}$, and that $A, B>0$, while $C, D<0$. Also observe that $2 A D+B^{2}=0$ and $-C^{2} D=1$. Note that $\mathcal{M}_{\text {seed }}$ satisfies $\mathcal{M}_{\text {seed }}^{\natural}=\mathcal{M}_{\text {seed }}$, where the anti-homomorphism $\downarrow$ denotes a 'generalized transposition' that is not simply transposition, see [12] for details.

The monodromy matrix (3.2), which we call the seed monodromy matrix, can be deformed in different ways. If the resulting monodromy matrix has a canonical factorization, then, as explained in section 2, its factorization will yield a solution to the field equations of the theory. In [4], a specific deformation of (3.2) was considered that gave rise to the extremal Reissner-Nordstöm black hole solution mentioned above. This deformation of the monodromy matrix was implemented by the transformation

$$
\mathcal{M}(\omega)=g^{\natural} \mathcal{M}_{\text {seed }} g, \quad g=e^{N},
$$

with $N$ a constant nilpotent matrix which resulted in [4]

$$
\mathcal{M}(\omega)=\left(\begin{array}{ccc}
A / \omega^{2}+A_{1} / \omega+A_{2} & B / \omega+B_{2} & C \\
-B / \omega-B_{2} & D & 0 \\
C & 0 & 0
\end{array}\right), \quad \operatorname{det} \mathcal{M}=1,
$$

with non-vanishing constants $A_{1}, A_{2}, B_{2}$ satisfying

$$
B_{2}^{2}=-2 A_{2} D, \quad A_{1}=-\left(B_{2} B\right) / D
$$


Note that $A_{2}>0$. Taking $B_{2}>0$, this yields $A_{1}=2 \sqrt{A A_{2}}$, where we used $B=\sqrt{-2 A D}$. Then, using $A \sqrt{-D}=P Q$ and defining $h=\sqrt{A_{2} \sqrt{-D}}>0$, we obtain

$$
\mathcal{M}(\omega)=\left(\begin{array}{ccc}
H^{2}(\omega) / \sqrt{-D} & \sqrt{2} \sqrt{\sqrt{-D}} H(\omega) & -1 / \sqrt{-D} \\
-\sqrt{2} \sqrt{\sqrt{-D}} H(\omega) & D & 0 \\
-1 / \sqrt{-D} & 0 & 0
\end{array}\right),
$$

where

$$
H(\omega)=h+\frac{\sqrt{P Q}}{\omega} .
$$

The associated space-time solution describes an extremal Reissner-Nordstöm black hole with line element

$$
d s_{4}^{2}=-\frac{1}{H^{2}(r)} d t^{2}+H^{2}(r)\left(d r^{2}+r^{2}\left(d \theta^{2}+\sin ^{2} \theta d \phi^{2}\right)\right),
$$

supported by a constant scalar field $e^{-2 \Phi}=-D^{3 / 2}=Q / P$.

In the following, we consider a different deformation of $(3.2)$. We replace $(Q, P)$ in $(3.2)$ by

$$
Q \rightarrow Q+h_{1} \omega, \quad P \rightarrow P+h_{2} \omega,
$$

where we view $\left(h_{1}, h_{2}\right) \in \mathbb{R}^{2}$ as deformation parameters. We restrict to $h_{1}>0, h_{2}>0$ throughout. We then obtain the monodromy matrix $\mathcal{M}=\mathcal{M}^{\natural}$,

$$
\mathcal{M}(\omega)=\left(\frac{H_{2}}{H_{1}}\right)^{1 / 3}\left(\begin{array}{ccc}
H_{1} H_{2} & \sqrt{2} H_{1} & -1 \\
-\sqrt{2} H_{1} & -H_{1} / H_{2} & 0 \\
-1 & 0 & 0
\end{array}\right), \quad \operatorname{det} \mathcal{M}=1
$$

where

$$
H_{1}(\omega)=h_{1}+\frac{Q}{\omega}, \quad H_{2}(\omega)=h_{2}+\frac{P}{\omega} .
$$

This deformed monodromy matrix is of the type $\mathcal{M}(\omega)=f(\omega) \tilde{\mathcal{M}}(\omega)$, where $\tilde{\mathcal{M}}(\omega)$ is a matrix with rational entries, and $f(\omega)$ is the third root of a rational function. Thus, according to the theorem of section $2, \mathcal{M}(\omega)$ possesses a canonical factorization, which below we carry out explicitly.

We note that the deformed monodromy matrix (3.11) is not of the form $g^{\natural} \mathcal{M}_{\text {seed }} g$, with $g$ given by $g(\omega)=e^{N(\omega)}$, where $N(\omega)$ is a nilpotent, possibly $\omega$-dependent lower triangular matrix. As shown in [4], such a transformation would result in a matrix of the form (3.5), with $\omega$-dependent coefficients $A_{1}, A_{2}, B_{2}$. This does not reproduce (3.11). Whether there exists a $g(\omega)$ such that the transformation $g^{\natural}(\omega) \mathcal{M}_{\text {seed }}(\omega) g(\omega)$ reproduces (3.11) is an open question that we will not address here.

Since the deformed monodromy matrix (3.11) is not of the form $g^{\natural} \mathcal{M}_{\text {seed }} g$, with $g$ a constant matrix, the resulting space-time solution lies outside of the class of solutions considered in $[17,19,22]$. The resulting space-time solution, which has a Killing horizon, will be stationary whenever the combination $J=h_{1} P-h_{2} Q$ is non-vanishing. The near-horizon solution, however, will exhibit the behaviour of a static attractor. We proceed to explain this. 
Let us compare (3.11) with (3.7). They will agree when

$$
h_{1} P=h_{2} Q, \quad h=\sqrt{h_{1} h_{2}} .
$$

The condition $h=\sqrt{h_{1} h_{2}}$ is a normalization condition that we will pick in the following. Therefore, only when $h_{1} P=h_{2} Q$ does the associated space-time solution describe the static solution (3.9) that is supported by a constant dilaton field. When $h_{1} P \neq h_{2} Q$, the interpolating space-time solution will not any longer remain static, and the dilaton field will cease to be constant. The solution will acquire a complicated dependence on the angular coordinate $\theta$, as will be shown below. The four-dimensional line element associated with this solution takes the form

$$
d s_{4}^{2}=-e^{-\phi_{2}}\left(d t+\mathcal{A}_{\phi} d \phi\right)^{2}+e^{\phi_{2}}\left(e^{\psi}\left(d r^{2}+r^{2} d \theta^{2}\right)+r^{2} \sin ^{2} \theta d \phi^{2}\right),
$$

where the functions $\phi_{2}, \psi$ and the one-form $\mathcal{A}=\mathcal{A}_{\phi} d \phi$ depend on the coordinates $(r, \theta)$. When $h_{1} P=h_{2} Q, \phi_{2}$ becomes a function of $r$ only, while $\psi=0$ and $\mathcal{A}_{\phi}=0$, and the line element reduces to the one in (3.9). However, when $h_{1} P \neq h_{2} Q, \psi$ and $\mathcal{A}$ are not any longer zero. They become non-trivial functions of $(r, \theta)$ that are given in terms of series expansions in the parameter $\tilde{J}=J /\left(h_{1} h_{2}\right)$, where

$$
\tilde{J}=\tilde{P}-\tilde{Q},
$$

with $\tilde{Q}=Q / h_{1}, \tilde{P}=P / h_{2}$. We recall that $\tilde{Q}>0, \tilde{P}>0$.

To assess the impact of a non-vanishing $\tilde{J}$ on the solution, one may consider taking $\tilde{J}$ to be small and working to first order in $\tilde{J}$. In doing so, we find the following. Asymptotically, as $r \rightarrow \infty$, we have $e^{\phi_{2}} \rightarrow h^{2}, e^{\psi} \rightarrow 1$, while $\mathcal{A}_{\phi}=-J \cos \theta$. Thus, the asymptotic geometry is stationary, with a NUT parameter $J$. On the other hand, when approaching the Killing horizon at $r=0$, the dilaton field tends to the constant value $e^{2 \Phi}=P / Q$, while $\psi$ tends to zero and $e^{\phi_{2}}$ behaves as $Q P / r^{2}$. However, $\mathcal{A}_{\phi}$ tends to $J \cos \theta(1-\cos \theta)$, and therefore the near-horizon geometry does not have the isometries of $A d S_{2} \times S^{1}$ or $A d S_{2} \times S^{2}$ [20]. Nevertheless, as $r \rightarrow 0$, the dilaton exhibits the behaviour of a static attractor $[13,14]$ : due to the deep throat in the geometry, the dilaton field flows to a constant value that is entirely specified by the electric/magnetic charges.

Now we proceed with the canonical factorization of $\mathcal{M}(\omega)$ given in (3.11). To perform the factorization explicitly, we will use the vectorial factorization method mentioned in section 2. Inspection of (3.11) shows that the monodromy matrix has poles in the $\omega$-plane located at $\omega=0,-\tilde{P},-\tilde{Q}$. Using the spectral curve relation (2.7), these values correspond to the following values on the $\tau$-plane,

$$
\begin{aligned}
\tau_{0}^{ \pm} & =\frac{1}{\rho}\left(v \pm \sqrt{\rho^{2}+v^{2}}\right), \\
\tau_{\tilde{P}}^{ \pm} & =\frac{1}{\rho}\left(v+\tilde{P} \pm \sqrt{\rho^{2}+(v+\tilde{P})^{2}}\right) \\
\tau_{\tilde{Q}}^{ \pm} & =\frac{1}{\rho}\left(v+\tilde{Q} \pm \sqrt{\rho^{2}+(v+\tilde{Q})^{2}}\right) .
\end{aligned}
$$


We recall $\rho>0$, and we take $v \in \mathbb{R} \backslash\{0,-\tilde{P},-\tilde{Q}\}$, so that the values (3.16) are never on the unit circle in the $\tau$-plane. Note that $\tau_{0}^{+} \tau_{0}^{-}=-1$, and similarly for the other two pairs in (3.16). Thus, given any of the pairs in (3.16), one of the values lies inside, while the other lies outside of the unit circle. The values inside of the unit circle will be denoted by $\tau^{+}$, while the values outside of the unit circle will be denoted by $\tau^{-}$. Depending on the region in the $(\rho, v)$-plane, $\tau^{+}$may either correspond to the + branch or the - branch in (3.16). The coordinates $(\rho, v)$ are related to the coordinates $(r, \theta)$ by

$$
\rho=r \sin \theta, \quad v=r \cos \theta,
$$

where $r>0$ and $0<\theta<\pi$.

The monodromy matrix (3.11) is the product of a matrix $\tilde{\mathcal{M}}(\omega)$ with a scalar factor $f(\omega)$. Then, the canonical factorization of $\mathcal{M}(\omega)=M_{-}(\tau) M_{+}(\tau)$ is obtained ${ }^{2}$ by performing the canonical factorization of $f(\omega)=f_{-}(\tau) f_{+}(\tau)$ and of $\tilde{\mathcal{M}}(\omega)=\tilde{M}_{-}(\tau) \tilde{M}_{+}(\tau)$, so that $M_{-}(\tau)=f_{-}(\tau) \tilde{M}_{-}(\tau)$ and $M_{+}(\tau)=f_{+}(\tau) \tilde{M}_{+}(\tau)$. Note that $M_{+}(\tau)$ has to satisfy the normalization condition $M_{+}(\tau=0)=\mathbb{I}$.

The scalar factor $f(\omega)=\left(H_{2}(\omega) / H_{1}(\omega)\right)^{1 / 3}$ has the canonical factorization

$$
f_{-}(\tau)=\left(\frac{h_{2}}{h_{1}}\right)^{1 / 3}\left(\frac{\tau_{\tilde{P}}^{-}}{\tau_{\tilde{Q}}^{-}}\right)^{1 / 3}\left(\frac{\tau-\tau_{\tilde{P}}^{+}}{\tau-\tau_{\tilde{Q}}^{+}}\right)^{1 / 3}, \quad f_{+}(\tau)=\left(\frac{\tau_{\tilde{P}}^{+}}{\tau_{\tilde{Q}}^{+}}\right)^{1 / 3}\left(\frac{\tau-\tau_{\tilde{P}}^{-}}{\tau-\tau_{\tilde{Q}}^{-}}\right)^{1 / 3}
$$

where we imposed the normalization $f_{+}(\tau=0)=1$. Here, the branch cuts are the line segments connecting $\tau_{\tilde{P}}^{+}$with $\tau_{\tilde{Q}}^{+}$and $\tau_{\tilde{P}}^{-}$with $\tau_{\tilde{Q}}^{-}$, and we take $1^{1 / 3}=1$. We note

$$
f_{-}(\tau=\infty)=\left(\frac{h_{2}}{h_{1}}\right)^{1 / 3}\left(\frac{\tau_{\tilde{P}}^{-}}{\tau_{\tilde{Q}}^{\bar{Q}}}\right)^{1 / 3}
$$

Next, we factorize

$$
\tilde{\mathcal{M}}(\omega)=\left(\begin{array}{ccc}
H_{1} H_{2} & \sqrt{2} H_{1} & -1 \\
-\sqrt{2} H_{1} & -H_{1} / H_{2} & 0 \\
-1 & 0 & 0
\end{array}\right)
$$

by using the vectorial factorization method mentioned in section 2 , which is set up in the form

$$
\tilde{\mathcal{M}} \tilde{M}_{+}^{-1}=\tilde{M}_{-},
$$

and which consists in considering the columns $\phi_{+}$of $\tilde{M}_{+}^{-1}$ and the columns $\phi_{-}$of $\tilde{M}_{-}$and solving the associated vectorial factorization problem, i.e.

$$
\tilde{\mathcal{M}} \phi_{+}=\phi_{-},
$$

column by column. In doing so, we use a generalized version of Liouville's theorem, and impose the normalization condition $\tilde{M}_{+}(\tau=0)=\mathbb{I}$. We refer to appendix A for the details of the factorization.

\footnotetext{
${ }^{2}$ We suppress the dependency of $M_{ \pm}$on $(\rho, v)$ for notational simplicity.
} 
Having obtained the factorization $\mathcal{M}(\omega)=M_{-}(\tau) M_{+}(\tau)$, we extract the matrix $M(\rho, v)$ that contains the space-time information,

$$
M(\rho, v)=M_{-}(\tau=\infty)=g\left(\begin{array}{ccc}
m_{1} & m_{2} & -1 \\
-m_{2} & m_{3} & 0 \\
-1 & 0 & 0
\end{array}\right)
$$

where

$$
\begin{aligned}
g & =f_{-}(\tau=\infty)=\left(\frac{h_{2}}{h_{1}}\right)^{1 / 3}\left(\frac{\tau_{\tilde{P}}^{-}}{\tau_{\tilde{Q}}^{-}}\right)^{1 / 3} \\
m_{1} & =h_{1} h_{2}\left(1-\frac{2 \tilde{Q}}{\rho\left(\tau_{0}^{+}-\tau_{0}^{-}\right)}\right)\left(1-\frac{2 \tilde{P}}{\rho\left(\tau_{0}^{+}-\tau_{0}^{-}\right)}\right)-2 h_{1} h_{2} \frac{\left(\tau_{\tilde{Q}}^{+}-\tau_{\tilde{P}}^{+}\right)\left(\tau_{0}^{+}-\tau_{\tilde{P}}^{-}\right)\left(\tau_{0}^{+}-\tau_{\tilde{Q}}^{+}\right)}{\tau_{\tilde{Q}}^{+}\left(\tau_{0}^{+}-\tau_{0}^{-}\right)^{2}} \\
m_{2} & =\sqrt{2} h_{1}\left(1-\frac{2 \tilde{Q}}{\rho\left(\tau_{0}^{+}-\tau_{0}^{-}\right)}\right)-\sqrt{2} h_{1} \frac{\left(\tau_{\tilde{Q}}^{+}-\tau_{\tilde{P}}^{+}\right)\left(\tau_{0}^{+}-\tau_{\tilde{Q}}^{+}\right)}{\tau_{\tilde{Q}}^{+}\left(\tau_{0}^{+}-\tau_{0}^{-}\right)} \\
m_{3} & =-\frac{h_{1}}{h_{2}} \frac{\tau_{\tilde{Q}}^{-}}{\tau_{\tilde{P}}^{-}}=-\frac{h_{1}}{h_{2}}\left(1+\frac{\tau_{\tilde{Q}}^{-}-\tau_{\tilde{P}}^{-}}{\tau_{\tilde{P}}^{-}}\right)
\end{aligned}
$$

We note the relation

$$
g^{3} m_{3}=-1 .
$$

Next, we relate the line element (3.14) to $M(\rho, v)$. We begin with the warp factor $\psi$, which, when viewed as a function of $(\rho, v)$, is obtained by integrating (2.4),

$$
\begin{aligned}
& \partial_{\rho} \psi=\frac{1}{4} \rho g^{4}\left[\left(\partial_{\rho}\left(g m_{3}\right)\right)^{2}-\left(\partial_{v}\left(g m_{3}\right)\right)^{2}\right], \\
& \partial_{v} \psi=\frac{1}{2} \rho g^{4} \partial_{\rho}\left(g m_{3}\right) \partial_{v}\left(g m_{3}\right) .
\end{aligned}
$$

Taking mixed derivatives of these equations, it can be verified that they are consistent, and hence (3.26) can be integrated. Below we will solve (3.26) in terms of a formal series expansion in powers of $\tilde{J}$,

$$
\psi(\rho, v)=\sum_{n=2}^{\infty} \psi_{n}(\rho, v) \tilde{J}^{n}
$$

up to a constant term which we set to zero by imposing the normalization condition $\psi=0$ when $\tilde{J}=0$. Note that there is no linear term in $\tilde{J}$. The $\psi_{n}$ will be determined by expanding the right hand side of (3.26) in powers of $\tilde{J}$, and integrating these equations order by order in $\tilde{J}$. We will then verify that when $\tilde{J}$ is small, it suffices to keep the first few terms in the series (3.27) to obtain an expression for $\psi$ that is excellent agreement with the exact solution of (3.26).

To relate the warp factor $e^{-\phi_{2}}$, the one-form $\mathcal{A}$ and the dilaton field $e^{-2 \Phi}$ to the data contained in $M(\rho, v)$, we use the following parametrization of $M(\rho, v)$ as a coset representative of $\operatorname{SL}(3, \mathbb{R}) / \mathrm{SO}(2,1)[12]$,

$$
M(\rho, v)=\left(\begin{array}{ccc}
e^{2 \Sigma_{1}} & e^{2 \Sigma_{1}} \chi_{2} & e^{2 \Sigma_{1}} \chi_{3} \\
-e^{2 \Sigma_{1}} \chi_{2} & -e^{2 \Sigma_{1}} \chi_{2}^{2}+e^{2 \Sigma_{2}} & -e^{2 \Sigma_{1}} \chi_{2} \chi_{3}+e^{2 \Sigma_{2}} \chi_{1} \\
e^{2 \Sigma_{1}} \chi_{3} & e^{2 \Sigma_{1}} \chi_{2} \chi_{3}-e^{2 \Sigma_{2}} \chi_{1} & -e^{2 \Sigma_{2}} \chi_{1}^{2}+e^{2 \Sigma_{1}} \chi_{3}^{2}+e^{2 \Sigma_{3}}
\end{array}\right)
$$


where

$$
\begin{aligned}
& \Sigma_{1}=\frac{1}{2}\left(\frac{1}{\sqrt{3}} \phi_{1}+\phi_{2}\right), \\
& \Sigma_{2}=-\frac{1}{\sqrt{3}} \phi_{1}, \\
& \Sigma_{3}=\frac{1}{2}\left(\frac{1}{\sqrt{3}} \phi_{1}-\phi_{2}\right),
\end{aligned}
$$

which satisfies $\Sigma_{1}+\Sigma_{2}+\Sigma_{3}=0$. Then

$$
\phi_{2}=2 \Sigma_{1}+\Sigma_{2}
$$

while the two-form $\mathcal{F}=d \mathcal{A}$ is determined by

$$
-e^{-2 \phi_{2}} * \mathcal{F}=d \chi_{3}-\chi_{1} d \chi_{2} .
$$

Here, the dual $*$ is with respect to the three-dimensional metric (2.1). The dilaton field is given by $e^{-2 \Phi}=e^{-\sqrt{3} \phi_{1} / 2}=e^{3 \Sigma_{2} / 2}$.

Comparing (3.28) with (3.23), we infer

$$
\begin{aligned}
e^{2 \Sigma_{1}} & =m_{1} g, \quad e^{2 \Sigma_{2}}=\left(m_{3}+\left(m_{2}\right)^{2} / m_{1}\right) g, \quad e^{-2 \Sigma_{3}}=\left(m_{1} m_{3}+\left(m_{2}\right)^{2}\right) g^{2}, \\
\chi_{1} & =-m_{2} /\left(m_{1} m_{3}+\left(m_{2}\right)^{2}\right), \quad \chi_{2}=m_{2} / m_{1}, \quad \chi_{3}=-1 / m_{1} .
\end{aligned}
$$

This results in the expressions

$$
\begin{aligned}
e^{2 \phi_{2}} & =g^{3} m_{1}\left(m_{1} m_{3}+\left(m_{2}\right)^{2}\right), \\
e^{-2 \Phi} & =g^{3 / 2}\left(m_{3}+\frac{\left(m_{2}\right)^{2}}{m_{1}}\right)^{3 / 2}, \\
d \chi_{3}-\chi_{1} d \chi_{2} & =\frac{1}{m_{1}\left(m_{1} m_{3}+\left(m_{2}\right)^{2}\right)}\left(m_{3} d m_{1}+m_{2} d m_{2}\right), \\
* \mathcal{F} & =d m_{1}+\frac{m_{2}}{m_{3}} d m_{2} .
\end{aligned}
$$

It can be verified that $d *\left(d m_{1}+\frac{m_{2}}{m_{3}} d m_{2}\right)=0$, so that $d \mathcal{F}=0$, and hence $\mathcal{F}=d \mathcal{A}$, locally.

Finally, we recall that the solution carries electric-magnetic charges $(Q, P)$, and hence is supported by an electric-magnetic field, which is described by a one-form $A^{0}$ that can be read off by performing the dimensional reduction of Einstein gravity in five dimensions to four dimensions [12], and given by

$$
A^{0}=\chi_{1} d t+A_{\phi} d \phi
$$

with $A_{\phi}$ determined by

$$
\begin{aligned}
\partial_{\rho} A_{\phi} & =-e^{2\left(\Sigma_{1}-\Sigma_{2}\right)} \rho \partial_{v} \chi_{2}+\mathcal{A}_{\phi} \partial_{\rho} \chi_{1}, \\
\partial_{v} A_{\phi} & =e^{2\left(\Sigma_{1}-\Sigma_{2}\right)} \rho \partial_{\rho} \chi_{2}+\mathcal{A}_{\phi} \partial_{v} \chi_{1} .
\end{aligned}
$$




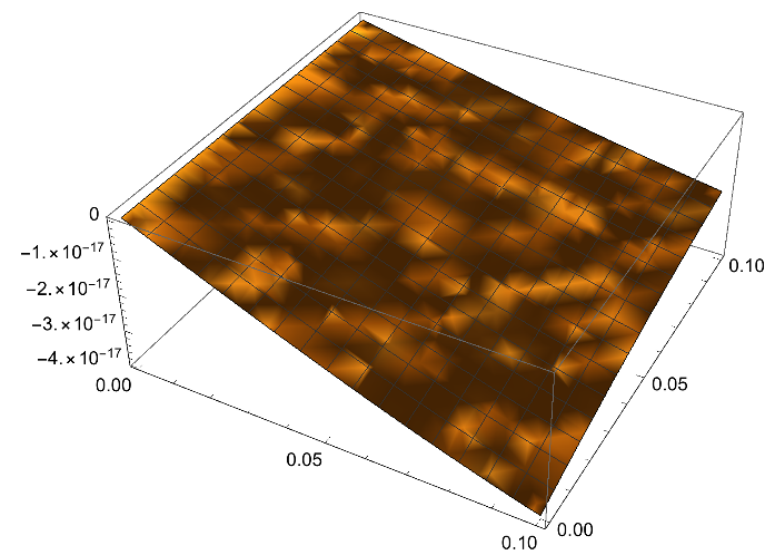

Figure 1. Behaviour near $\rho=0, v=0$, in the range $0 \leq \rho \leq 0.1,0 \leq v \leq 0.1$.

Now we turn to the interpretation of the space-time solution described by line element (3.14), by (3.33), (3.35) and by (3.26). To this end, and for concreteness, we focus on the region $\rho>0, v>0$, so that also $v+\tilde{Q}>0, v+\tilde{P}>0$. Then,

$$
\begin{aligned}
& \rho \tau_{0}^{+}=v-\sqrt{v^{2}+\rho^{2}}, \\
& \rho \tau_{\tilde{Q}}^{+}=v+\tilde{Q}-\sqrt{(v+\tilde{Q})^{2}+\rho^{2}}, \\
& \rho \tau_{\tilde{P}}^{+}=v+\tilde{P}-\sqrt{(v+\tilde{P})^{2}+\rho^{2}} .
\end{aligned}
$$

First we consider the series expansion (3.27). As we mentioned below (3.27), we determine the explicit form of the $\psi_{n}$ by expanding the right hand side of (3.26) in powers of $\tilde{J}$, and subsequently integrating these equations order by order in $\tilde{J}$. In this way, we find that the first terms in this series, which begins with $n=2$, are given by

$$
\begin{aligned}
\psi_{2}(\rho, v) & =-\frac{\rho^{2}}{18\left[(\tilde{Q}+v)^{2}+\rho^{2}\right]^{2}}, \\
\psi_{3}(\rho, v) & =\frac{\rho^{2}(\tilde{Q}+v)}{9\left[(\tilde{Q}+v)^{2}+\rho^{2}\right]^{3}}, \\
\psi_{4}(\rho, v) & =\frac{\rho^{2}\left[-24(\tilde{Q}+v)^{2}+5 \rho^{2}\right]}{144\left[(\tilde{Q}+v)^{2}+\rho^{2}\right]^{4}} .
\end{aligned}
$$

We now verify that if we only keep these first terms in the series expansion (3.27), the resulting function $\psi=\sum_{n=2}^{4} \psi_{n}(\rho, v) \tilde{J}^{n}$ is in excellent agreement with the exact solution of (3.26) for small values of $\tilde{J}$. We do this by comparing the expression on the left hand side of (3.26), computed using $\psi=\sum_{n=2}^{4} \psi_{n}(\rho, v) \tilde{J}^{n}$, with the exact expression on the right hand side of (3.26). This is depicted in figures 1 and 2 for various ranges of $(\rho, v)$, where we plotted the difference of the two expressions appearing in the first equation of (3.26) for the values $\tilde{Q}=1, \tilde{J}=0.001$. 


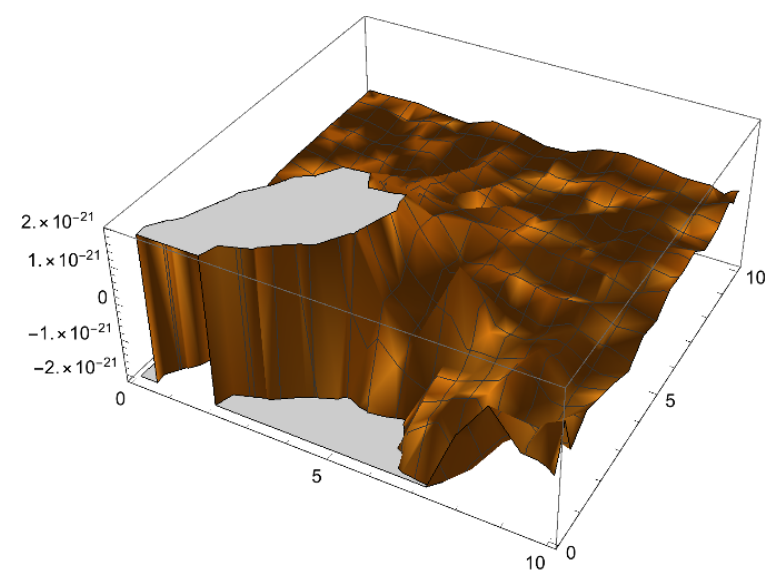

Figure 2. Behaviour in the range $0.1 \leq \rho \leq 10,0.1 \leq v \leq 10$.

In the following, we work at first order in $\tilde{J}$, in which case $\psi$ may be approximated by $\psi=0$. Then, from (3.33) we infer

$$
\mathcal{A}_{\phi}=-J \frac{\left(\rho^{2}+v(\tilde{Q}+v)\right)\left(v-\sqrt{\rho^{2}+v^{2}}\right)}{\left(\rho^{2}+v^{2}\right) \sqrt{\rho^{2}+(\tilde{Q}+v)^{2}}}+\mathcal{O}\left(\tilde{J}^{2}\right)
$$

up to a constant, while from (3.35) we obtain

$$
A_{\phi}=\sqrt{2} h_{2}\left[\left(\tilde{Q}+\frac{\tilde{J}}{2}\right) \frac{v}{\sqrt{\rho^{2}+v^{2}}}+\frac{\tilde{J}}{2} \frac{\sqrt{\rho^{2}+(\tilde{Q}+v)^{2}}\left(v-\sqrt{\rho^{2}+v^{2}}\right)}{\rho^{2}+v^{2}+\tilde{Q} \sqrt{\rho^{2}+v^{2}}}\right]+\mathcal{O}\left(\tilde{J}^{2}\right)
$$

up to a constant.

To assess the impact of a non-vanishing $\tilde{J}$ on the solution, we switch to coordinates $(r, \theta)$ given by (3.17). Asymptotically, as $r \rightarrow \infty$, we have $e^{-2 \Phi} \rightarrow h_{1} / h_{2}, e^{\phi_{2}} \rightarrow$ $h_{1} h_{2}, e^{\psi}=1$, while $\mathcal{A}_{\phi}=-J \cos \theta$ and $A_{\phi}=\sqrt{2} P \cos \theta$, up to a constant. Thus, the asymptotic solution is stationary, with a NUT parameter $J$, and is reminiscent of four-dimensional extremal solutions with NUT charge $[23,24]$ that are related to fivedimensional extremal solutions through the $4 \mathrm{~d} / 5 \mathrm{~d}$ lift [25].

On the other hand, when approaching the Killing horizon $r=0$, the dilaton field tends to the constant value $e^{2 \Phi} \rightarrow P / Q$, while $e^{\phi_{2}}$ tends to $Q P / r^{2}, A_{\phi}$ tends to $\sqrt{2} P \cos \theta$ and $\mathcal{A}_{\phi}$ tends to $J \cos \theta(1-\cos \theta)$. Hence, the resulting geometry does not have the isometries of $A d S_{2} \times S^{2}$. Nevertheless, the dilaton behaves like a static attractor with charges $(Q, P)$ : the dilaton flows to a value entirely determined in terms of these charges, and the area of the Killing horizon at $r=0$ (which has zero angular velocity) equals $4 \pi Q P$, and is thus also determined in terms of the charges. The Kretschmann scalar and $R^{\mu \nu} R_{\mu \nu}$ are finite at $r=0$. This static attractor behaviour at $r \rightarrow 0$ is then an unusual feature of this stationary solution. It can be verified that corrections of order $\tilde{J}^{2}$ do not affect the attractor behaviour just described. 
The behaviour just described may raise the following question. The canonical factorization of $\mathcal{M}(\omega)$ yields factors $M_{ \pm}(\tau, \rho, v)$, from which one extracts the space-time information $M(\rho, v)$ by means of $M(\rho, v)=M_{-}(\tau=\infty, \rho, v)$. Now consider the coordinates $(r, \theta)$ given in (3.17). Since asymptotically, as $r \rightarrow \infty$, the matrix $M(\rho, v)$ tends to a constant matrix, this may naively suggest that asymptotically, space-time is static and flat. This naive expectation is, however, incorrect. To obtain the asymptotic form of the space-time line element, one also needs to calculate the one-form $\mathcal{A}$ that appears in the line element (3.14). This is done by first calculating the field strength $\mathcal{F}=d \mathcal{A}$, whose components are expressed in terms of derivatives of the entries of $M(\rho, v)$, see (3.33). To first order in $\tilde{J}, \mathcal{F}$ exhibits an asymptotic falloff of the form $J / r$. The associated potential $\mathcal{A}_{\phi}$ is thus non-vanishing, $\mathcal{A}_{\phi}=-J \cos \theta$.

Next, we verify the validity of the substitution rule. As mentioned in section 2 , this is done by considering $M(\rho, v)$ in the limit $\rho \rightarrow 0^{+}$, and subsequently setting $v=\omega$. If the resulting matrix equals $\mathcal{M}(\omega)$, the substitution rule holds in the example under study. To take the limit $\rho \rightarrow 0^{+}$, we need to specify a region in parameter space $(\rho, v)$. We focus again on the region $v>0$, for concreteness. We recall that $\tilde{Q}>0, \tilde{P}>0$. Then, using (3.36), we obtain in the limit $\rho \rightarrow 0^{+}$,

$$
\begin{array}{rlrl}
\tau_{0}^{+} & =-\frac{\rho}{2 v}, & \tau_{0}^{-}=\frac{2 v}{\rho}, \\
\tau_{\tilde{Q}}^{+}=-\frac{\rho}{2(v+\tilde{Q})}, & \tau_{\tilde{Q}}^{-}=\frac{2(v+\tilde{Q})}{\rho}, \\
\tau_{\tilde{P}}^{+}=-\frac{\rho}{2(v+\tilde{P})}, & \tau_{\tilde{P}}^{-}=\frac{2(v+\tilde{P})}{\rho} .
\end{array}
$$

Using this, we infer

$$
\lim _{\rho \rightarrow 0^{+}} M(\rho, v)=\mathcal{M}(v),
$$

thus verifying the validity of the substitution rule in the region $v>0$.

Finally, we address the following question: the monodromy matrix (3.11) depends on the parameters $h_{1}, h_{2}$. When factorizing, the factors $M_{-}$and $M_{+}$will also depend on these parameters. Is this dependence a continuous one? This is not entirely obvious. Consider, for instance the limit $h_{2} \rightarrow 0$ : the zero $\omega=-P / h_{2}$ of $H_{2}$ in (3.11) will move to infinity. We will verify that the factorization of (3.11) with $h_{2}=0$ gives results that coincide with those obtained by factorizing (3.11) with $h_{2} \neq 0$ and subsequently taking the limit $h_{2} \rightarrow 0$.

Let us first consider the case when $h_{1} \rightarrow 0, h_{2} \rightarrow 0$. We consider the region $v>0$, and we recall $Q / h_{1}>0, P / h_{2}>0$. In the limit $h_{1} \rightarrow 0, h_{2} \rightarrow 0$, we have

$$
\tau_{\tilde{Q}}^{+} \rightarrow-\frac{\rho h_{1}}{2 Q} \rightarrow 0, \quad \frac{\tau_{\tilde{Q}}^{+}}{h_{1}} \rightarrow-\frac{\rho}{2 Q}, \quad h_{1} \tau_{\tilde{Q}}^{-} \rightarrow \frac{2 Q}{\rho},
$$

and similarly for $P$. We obtain

$$
A=\frac{4 Q P}{\rho^{2}\left(\tau_{0}^{+}-\tau_{0}^{-}\right)^{2}}, \quad B=-2 \sqrt{2} \frac{Q}{\rho\left(\tau_{0}^{+}-\tau_{0}^{-}\right)}, \quad C=-\frac{Q}{P},
$$

in agreement with [4]. 
Next, we keep $h_{1} \neq 0$, and send $h_{2} \rightarrow 0$. We again consider the region $v>0$, with $Q / h_{1}>0, P / h_{2}>0$. We obtain

$$
\begin{aligned}
A & =\left(h_{1}-\frac{2 Q}{\rho\left(\tau_{0}^{+}-\tau_{0}^{-}\right)}\right)\left(-\frac{2 P}{\rho\left(\tau_{0}^{+}-\tau_{0}^{-}\right)}\right)+4 h_{1} P \frac{\left(\tau_{0}^{+}-\tau_{\tilde{Q}}^{+}\right)}{\rho\left(\tau_{0}^{+}-\tau_{0}^{-}\right)^{2}}=-2 h_{1} P \frac{\left(\tau_{\tilde{Q}}^{+}-\tau_{\tilde{Q}}^{-}\right)}{\rho\left(\tau_{0}^{+}-\tau_{0}^{-}\right)^{2}}, \\
B & =\sqrt{2} h_{1} \frac{\left[\tau_{0}^{+}-\tau_{\tilde{Q}}^{-}\right]}{\left(\tau_{0}^{+}-\tau_{0}^{-}\right)}, \\
C & =-\frac{h_{1}}{2 P} \rho \tau_{\tilde{Q}}^{-} .
\end{aligned}
$$

Comparing with the explicit factorization results that we obtain when factorizing (3.11) with $h_{2}=0$, we find perfect agreement. Thus, we have verified that the factorization depends in a continuous manner on the parameter $h_{2}$ : there is no breakdown when $h_{2}=0$ (keeping $h_{1} \neq 0$ ).

\subsection{Deformed monodromy matrices in dimensionally reduced Einstein gravity}

Next, we consider four-dimensional Einstein gravity reduced to two space-like dimensions. The associated monodromy matrices are $2 \times 2$ matrices that satisfy

$$
\mathcal{M}=\mathcal{M}^{\natural}=\mathcal{M}^{T}, \quad \operatorname{det} \mathcal{M}=1,
$$

where, is this case, the operation $\downarrow$ is transposition. The monodromy matrix is thus a symmetric matrix, which we write in the form

$$
\mathcal{M}=\left(\begin{array}{cc}
b & a \\
a & b \\
R^{2}
\end{array}\right)
$$

The monodromy matrix depends on three continuous functions $a, b$ and $R^{2}$, with $b^{2} R^{2}-a^{2}=$ 1 on $\Gamma_{\omega} . \mathcal{M}$ can be decomposed as

$$
\mathcal{M}=\Sigma D \Sigma^{-1} J
$$

where

$$
\Sigma=\left(\begin{array}{cc}
1 & 1 \\
R & -R
\end{array}\right), \quad D=\left(\begin{array}{cc}
a+b R & 0 \\
0 & a-b R
\end{array}\right), \quad J=\left(\begin{array}{ll}
0 & 1 \\
1 & 0
\end{array}\right) .
$$

Here we assumed $R \neq 0$, so that $\Sigma$ is invertible,

$$
\Sigma^{-1}=\frac{1}{2 R}\left(\begin{array}{cc}
R & 1 \\
R & -1
\end{array}\right) .
$$

Note that the case $R \equiv 0$ requires $a$ to be imaginary (since $\operatorname{det} \mathcal{M}=1$ ). We discard this case in the following.

Now let us discuss the canonical factorization of (3.47). The monodromy matrix (3.46) depends on three functions $a, b$ and $R^{2}$ that, in the decomposition (3.47), get assembled into combinations $a \pm b R$ and $R$. To proceed, we have to make a choice for the functions 
$R$. We take $R$ to be a rational function of $\omega$ that is bounded at $\omega=\infty$, with no zeroes and no poles on $\Gamma_{\omega}$, and similarly for its inverse $R^{-1}$. Note that the Schwarzschild solution is captured by this class of functions $R$. For this class of functions $R$, the combinations $a \pm b R$ are continuous functions of $\omega$ and non-vanishing on $\Gamma_{\omega}$, and hence possess a canonical factorization according to the theorem in section 2 . Now let us discuss the various factors in the decomposition (3.47):

1. The diagonal matrix $D=\operatorname{diag}\left(d_{1}, d_{2}\right)$, which is determined in terms of the combinations $d_{1}=a+b R, d_{2}=a-b R$, has a canonical factorization, $D=D_{-} D_{+}$,

$$
D_{-}=\left(\begin{array}{cc}
d_{1-} & 0 \\
0 & d_{2-}
\end{array}\right), \quad D_{+}=\left(\begin{array}{cc}
d_{1+} & 0 \\
0 & d_{2+}
\end{array}\right) .
$$

Since $D$ is diagonal, this is a scalar factorization problem.

2. The functions $R$ and $R^{-1}$ are rational functions of $\omega$ that are bounded at $\omega=\infty$. We normalize $R(\infty)=1$. Writing $R(\omega)=r(\omega) / s(\omega)$, we take $r(\omega)$ and $s(\omega)$ to be both polynomials of degree $n$. Then, using the algebraic curve (2.6), the resulting function $R(\omega(\tau))$ and its inverse will be rational functions in $\tau$ with $2 n$ poles and $2 n$ zeroes (counting multiplicities). For concreteness, we take $R(\omega(\tau))$ to have $2 n$ simple poles located at $\tau_{i}^{ \pm}(i=1, \ldots, n)$. The $n$ poles at $\tau_{i}^{+}$are located in the interior of the unit disc, and the $n$ poles $\tau_{i}^{-}$are located in the exterior of the unit disc in the $\tau$ plane. Thus, we take $R(\omega(\tau))$ to be

$$
R(\omega(\tau))=\frac{q(\tau)}{\prod_{i=1}^{n}\left(\tau-\tau_{i}^{+}\right)\left(\tau-\tau_{i}^{-}\right)},
$$

where $q(\tau)$ denotes a polynomial of degree $2 n$ (with $n$ simple zeroes inside the unit circle, and $n$ simple zeroes outside the unit circle), satisfying the normalization condition $q(\tau)=\tau^{2 n}$ at $\tau=\infty$, so that $R(\omega(\infty))=1$.

Next, let us discuss the canonical factorization ${ }^{3}$ of $\mathcal{M}(\omega)$,

$$
\mathcal{M}(\omega(\tau))=M_{-}(\tau) M_{+}(\tau), \quad \tau \in \Gamma .
$$

We begin by noting that (3.52) can be written as

$$
D_{+} \Sigma^{-1} J M_{+}^{-1}=D_{-}^{-1} \Sigma^{-1} M_{-} .
$$

Denoting

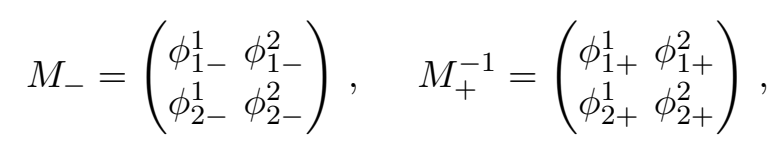

we obtain

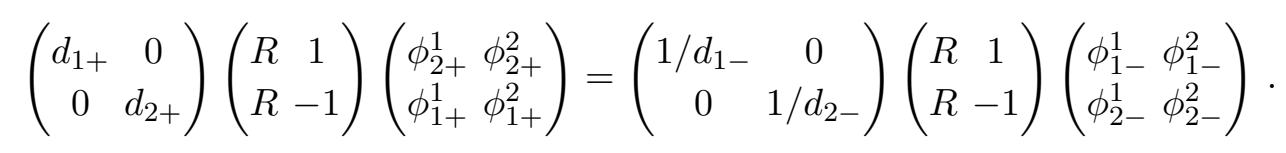

\footnotetext{
${ }^{3}$ We suppress the dependency of $M_{ \pm}$on $(\rho, v)$ for notational simplicity.
} 
This yields the following pair of linear systems $\left(S_{1}\right.$ and $\left.S_{2}\right)$,

$$
\begin{aligned}
& S_{1}:\left\{\begin{array}{l}
R d_{1+} \phi_{2+}^{1}+d_{1+} \phi_{1+}^{1}=\left(R \phi_{1-}^{1}+\phi_{2-}^{1}\right) / d_{1-}, \\
R d_{2+} \phi_{2+}^{1}-d_{2+} \phi_{1+}^{1}=\left(R \phi_{1-}^{1}-\phi_{2-}^{1}\right) / d_{2-},
\end{array}\right. \\
& S_{2}:\left\{\begin{array}{l}
R d_{1+} \phi_{2+}^{2}+d_{1+} \phi_{1+}^{2}=\left(R \phi_{1-}^{2}+\phi_{2-}^{2}\right) / d_{1-}, \\
R d_{2+} \phi_{2+}^{2}-d_{2+} \phi_{1+}^{2}=\left(R \phi_{1-}^{2}-\phi_{2-}^{2}\right) / d_{2-} .
\end{array}\right.
\end{aligned}
$$

Here we have separated the functions that are analytic inside the unit disc in the $\tau$-plane from those that are analytic in the outside region of the unit disc. The only exception to this is the function $R$. By assumption, $R(\omega(\tau))$ is a rational function satisfying $R(\omega(\infty))=1$, with $2 n$ simple zeroes and $2 n$ simple poles. Then, by means of a generalization of Liouville's theorem (see the lemma on page 14 of [4]), we have

$$
\begin{array}{r}
S_{1}:\left\{\begin{array}{l}
R d_{1+} \phi_{2+}^{1}+d_{1+} \phi_{1+}^{1}=\left(R \phi_{1-}^{1}+\phi_{2-}^{1}\right) / d_{1-}=\frac{p_{1}(\tau)}{\prod_{i=1}^{n}\left(\tau-\tau_{i}^{+}\right)\left(\tau-\tau_{i}^{-}\right)}, \\
R d_{2+} \phi_{2+}^{1}-d_{2+} \phi_{1+}^{1}=\left(R \phi_{1-}^{1}-\phi_{2-}^{1}\right) / d_{2-}=\frac{p_{2}(\tau)}{\prod_{i=1}^{n}\left(\tau-\tau_{i}^{+}\right)\left(\tau-\tau_{i}^{-}\right)},
\end{array}\right. \\
S_{2}:\left\{\begin{array}{l}
R d_{1+} \phi_{2+}^{2}+d_{1+} \phi_{1+}^{2}=\left(R \phi_{1-}^{2}+\phi_{2-}^{2}\right) / d_{1-}=\frac{p_{3}(\tau)}{\prod_{i=1}^{n}\left(\tau-\tau_{i}^{+}\right)\left(\tau-\tau_{i}^{-}\right)}, \\
R d_{2+} \phi_{2+}^{2}-d_{2+} \phi_{1+}^{2}=\left(R \phi_{1-}^{2}-\phi_{2-}^{2}\right) / d_{2-}=\frac{p_{4}(\tau)}{\prod_{i=1}^{n}\left(\tau-\tau_{i}^{+}\right)\left(\tau-\tau_{i}^{-}\right)},
\end{array}\right.
\end{array}
$$

where $p_{j}(\tau), j=1,2,3,4$ are polynomials of degree $2 n$ in $\tau$, to ensure boundedness at $\tau=\infty$. They contain a total of $8 n+4$ constants. These constants are determined by

1. imposing the normalization condition $M_{+}(\tau=0)=\mathbb{I}$, which yields the four normalization conditions

$$
\phi_{1+}^{1}(\tau=0)=1, \phi_{2+}^{2}(\tau=0)=1, \phi_{2+}^{1}(\tau=0)=0, \phi_{1+}^{2}(\tau=0)=0 ;
$$

2. imposing that the $\phi_{i \pm}^{j}$ have appropriate analyticity properties at the poles and zeroes of $R(\omega(\tau))$. This yields $8 n$ conditions.

Thus, in total, there are $8 n+4$ conditions. They will uniquely determine the value of the $8 n+4$ constants, provided that these $8 n+4$ conditions, written as a linear system for the $8 n+4$ constants, form a linear system whose determinant is different from zero. Then, solving the linear systems $S_{1,2}$ results in explicit expressions for $M_{ \pm}(\tau)$ in (3.52). There may exist points/curves in the $(\rho, v)$ plane where the determinant is zero, in which case there is a breakdown of canonical factorizability at these locations. Note that since we are dealing with linear systems, we are guaranteed to be able to deduce when $\mathcal{M}$ is canonically factorizable, and when not.

In the above, we took $R$ to have only simple zeroes and simple poles. We can easily generalise the above discussion to the case when $R$ contains zeroes/poles of higher order. Using Liouville's theorem, higher order poles can be dealt with in a similar manner as with simple poles. 
The explicit solution to the systems $S_{1,2}$ determines the explicit form of $M_{ \pm}$. The solution to Einstein's field equations is then read off from

$$
M(\rho, v)=M_{-}(\tau=\infty) .
$$

Let us therefore determine $M_{-}(\tau=\infty)$. Denoting $\left[p_{j} / \tau^{2 n}\right]_{\left.\right|_{\tau=\infty}}=A_{j}$ and using $R(\omega(\infty))=$ 1 , we obtain

$$
\begin{aligned}
M(\rho, v) & =\left(\begin{array}{ll}
\phi_{1-}^{1}(\infty) & \phi_{1-}^{2}(\infty) \\
\phi_{2-}^{1}(\infty) & \phi_{2-}^{2}(\infty)
\end{array}\right) \\
& =\frac{1}{2}\left(\begin{array}{ll}
d_{1-}(\infty) A_{1}+d_{2-}(\infty) A_{2} & d_{1-}(\infty) A_{3}+d_{2-}(\infty) A_{4} \\
d_{1-}(\infty) A_{1}-d_{2-}(\infty) A_{2} & d_{1-}(\infty) A_{3}-d_{2-}(\infty) A_{4}
\end{array}\right),
\end{aligned}
$$

where $d_{1-}, d_{2-}$ are evaluated at $\tau=\infty$. The constants $A_{1}, A_{2}, A_{3}, A_{4}$ are not all independent. The constants $A_{3}$ and $A_{4}$ are related to $A_{1}$ and $A_{2}$, as follows. Recall that $M(\rho, v)$ satisfies $M=M^{\natural}=M^{T}$, which implies

$$
d_{1-}(\infty) A_{3}+d_{2-}(\infty) A_{4}=d_{1-}(\infty) A_{1}-d_{2-}(\infty) A_{2} .
$$

In addition, since $\operatorname{det} M=1(M \in \mathrm{SL}(2, \mathbb{R}) / \mathrm{SO}(2)), M$ has the form

$$
M=\left(\begin{array}{cc}
\Delta+B^{2} / \Delta & B / \Delta \\
B / \Delta & \Delta^{-1}
\end{array}\right)
$$

This results in

$$
d_{1-}(\infty) A_{3}-d_{2-}(\infty) A_{4}=4 \frac{\left(1+\frac{1}{4}\left(d_{1-}(\infty) A_{1}-d_{2-}(\infty) A_{2}\right)^{2}\right)}{d_{1-}(\infty) A_{1}+d_{2-}(\infty) A_{2}}
$$

Then, $M$ in (3.60) can be expressed in terms of the constants $A_{1}$ and $A_{2}$.

Let us return to (3.46): the functions $a$ and $b$ are continuous on $\Gamma_{\omega}$, and $a \pm b R$ are non-vanishing on $\Gamma_{\omega}$, as required for monodromy matrices; the only extra condition imposed in the above was that $R$ is a rational function of $\omega$, as in the Schwarzschild case. Once $R$ is chosen, we still have the freedom to pick $a$ and $b$ from a broad class of functions. Each such choice will, upon canonical factorization, result in a solution to Einstein's field equations. Thus, by changing $a$ and $b$ we obtain a large class of solutions to the gravitational field equations. Note that small changes in $a$ and $b$ may result in highly non-trivial changes of the space-time solution. We illustrate this in the next subsubsection by picking combinations $a \pm b R$ that possess an essential singularity at $\omega=0$ and depend on a free parameter $\xi$. This describes a deformation of the monodromy matrix associated with the Schwarzschild solution. The canonical factorization of this deformed monodromy matrix yields a complicated space-time metric.

\subsubsection{Deforming the monodromy matrix of the Schwarzschild solution}

Let us now consider a concrete example. We take

$$
R(\omega)=\frac{\omega+m}{\omega-m}, \quad a(\omega)=\sinh \frac{\xi}{\omega}, \quad b(\omega) R(\omega)=\cosh \frac{\xi}{\omega},
$$


where $m \in \mathbb{R}, \xi \in \mathbb{R}$. When $\xi=0$, the resulting monodromy matrix $\mathcal{M}(\omega)$ is the one associated to the Schwarzschild solution [7] in the region $v>m>0, \rho>0$,

$$
\mathcal{M}_{\text {Schwarzschild }}=\left(\begin{array}{cc}
R^{-1} & 0 \\
0 & R
\end{array}\right) \text {. }
$$

When $\xi \neq 0$, the monodromy matrix represents a deformation of $\mathcal{M}_{\text {Schwarzschild, }}$ with the property that at $\omega \rightarrow \infty, \mathcal{M}$ approaches $\mathbb{I}$, which corresponds to flat space-time. As soon as $\xi \neq 0$, the monodromy matrix ceases to be diagonal. By picking a deformation such that $a$ and $b R$ are given in terms of exponentials of rational functions of $\omega$, we insure that the matrix $D$ can be easily factorized canonically. The diagonal matrix $D$ has entries

$$
d_{1}=e^{\frac{\xi}{\omega}}, \quad d_{2}=-\frac{1}{d_{1}}=-e^{-\frac{\xi}{\omega}} .
$$

Using (3.69), we obtain

$$
\begin{array}{rr}
d_{1-}=e^{\frac{2 \xi \beta}{\rho\left(\tau-\tau_{0}^{+}\right)}}, & d_{1+}=e^{\frac{2 \xi \alpha}{\rho\left(\tau-\tau_{0}^{-}\right)}}, \\
d_{2-}=\frac{1}{d_{1-}}=e^{-\frac{2 \xi \beta}{\rho\left(\tau-\tau_{0}^{+}\right)}}, & d_{2+}=-\frac{1}{d_{1+}}=-e^{-\frac{2 \xi \alpha}{\rho\left(\tau-\tau_{0}^{-}\right)}},
\end{array}
$$

where we made a choice of signs, and where we introduced

$$
\alpha=\frac{\tau_{0}^{-}}{\tau_{0}^{+}-\tau_{0}^{-}}, \quad \beta=-\frac{\tau_{0}^{+}}{\tau_{0}^{+}-\tau_{0}^{-}} .
$$

The values $\tau_{0}^{ \pm}$are the two values associated to $\omega=0$ through the algebraic curve (2.6),

$$
\omega=-\frac{\rho}{2} \frac{\left(\tau-\tau_{0}^{+}\right)\left(\tau-\tau_{0}^{-}\right)}{\tau} .
$$

They are given by (recall that $\rho>0$ )

$$
\tau_{0}^{ \pm}=\frac{v \pm \sqrt{v^{2}+\rho^{2}}}{\rho}
$$

Here, $\tau_{0}^{+}$denotes the value in the interior of the unit disc in the $\tau$ plane, while $\tau_{0}^{-}$denotes the value in the exterior of the unit disc. The choice of the sign in (3.70) is then correlated with the region in the $(\rho, v)$ plane. Similarly, we introduce the two values $\tau_{1}^{ \pm}$associated to $\omega=m$,

$$
\tau_{1}^{ \pm}=\frac{(v-m) \pm \sqrt{(v-m)^{2}+\rho^{2}}}{\rho}
$$

and the two values $\tau_{2}^{ \pm}$associated to $\omega=-m$,

$$
\tau_{2}^{ \pm}=\frac{(v+m) \pm \sqrt{(v+m)^{2}+\rho^{2}}}{\rho}
$$


Multiplying the two linear systems $S_{1,2}$ in (3.57) by $R^{-1}$, we obtain

$$
\begin{array}{r}
S_{1}:\left\{\begin{array}{l}
d_{1+} \phi_{2+}^{1}+R^{-1} d_{1+} \phi_{1+}^{1}=\left(\phi_{1-}^{1}+R^{-1} \phi_{2-}^{1}\right) / d_{1-}=\frac{A_{1} \tau^{2}+B_{1} \tau+C_{1}}{\left(\tau-\tau_{2}^{+}\right)\left(\tau-\tau_{2}^{-}\right)}, \\
d_{2+} \phi_{2+}^{1}-R^{-1} d_{2+} \phi_{1+}^{1}=\left(\phi_{1-}^{1}-R^{-1} \phi_{2-}^{1}\right) / d_{2-}=\frac{A_{2} \tau^{2}+B_{2} \tau+C_{2}}{\left(\tau-\tau_{2}^{+}\right)\left(\tau-\tau_{2}^{-}\right)}
\end{array}\right. \\
S_{2}:\left\{\begin{array}{l}
d_{1+} \phi_{2+}^{2}+R^{-1} d_{1+} \phi_{1+}^{2}=\left(\phi_{1-}^{2}+R^{-1} \phi_{2-}^{2}\right) / d_{1-}=\frac{A_{3} \tau^{2}+B_{3} \tau+C_{3}}{\left(\tau-\tau_{2}^{+}\right)\left(\tau-\tau_{2}^{-}\right)} \\
d_{2+} \phi_{2+}^{2}-R^{-1} d_{2+} \phi_{1+}^{2}=\left(\phi_{1-}^{2}-R^{-1} \phi_{2-}^{2}\right) / d_{2-}=\frac{A_{4} \tau^{2}+B_{4} \tau+C_{4}}{\left(\tau-\tau_{2}^{+}\right)\left(\tau-\tau_{2}^{-}\right)}
\end{array}\right.
\end{array}
$$

which results in

$$
\begin{aligned}
& \phi_{1+}^{1}=d_{1+} \frac{A_{2} \tau^{2}+B_{2} \tau+C_{2}}{2\left(\tau-\tau_{1}^{+}\right)\left(\tau-\tau_{1}^{-}\right)}-d_{2+} \frac{A_{1} \tau^{2}+B_{1} \tau+C_{1}}{2\left(\tau-\tau_{1}^{+}\right)\left(\tau-\tau_{1}^{-}\right)}, \\
& \phi_{1+}^{2}=d_{1+} \frac{A_{4} \tau^{2}+B_{4} \tau+C_{4}}{2\left(\tau-\tau_{1}^{+}\right)\left(\tau-\tau_{1}^{-}\right)}-d_{2+} \frac{A_{3} \tau^{2}+B_{3} \tau+C_{3}}{2\left(\tau-\tau_{1}^{+}\right)\left(\tau-\tau_{1}^{-}\right)}, \\
& \phi_{2+}^{1}=-d_{1+} \frac{A_{2} \tau^{2}+B_{2} \tau+C_{2}}{2\left(\tau-\tau_{2}^{+}\right)\left(\tau-\tau_{2}^{-}\right)}-d_{2+} \frac{A_{1} \tau^{2}+B_{1} \tau+C_{1}}{2\left(\tau-\tau_{2}^{+}\right)\left(\tau-\tau_{2}^{-}\right)}, \\
& \phi_{2+}^{2}=-d_{1+} \frac{A_{4} \tau^{2}+B_{4} \tau+C_{4}}{2\left(\tau-\tau_{2}^{+}\right)\left(\tau-\tau_{2}^{-}\right)}-d_{2+} \frac{A_{3} \tau^{2}+B_{3} \tau+C_{3}}{2\left(\tau-\tau_{2}^{+}\right)\left(\tau-\tau_{2}^{-}\right)}, \\
& \phi_{1-}^{1}=\left(\frac{1}{d_{1-}}\right) \frac{A_{2} \tau^{2}+B_{2} \tau+C_{2}}{2\left(\tau-\tau_{2}^{+}\right)\left(\tau-\tau_{2}^{-}\right)}+\left(\frac{1}{d_{2-}}\right) \frac{A_{1} \tau^{2}+B_{1} \tau+C_{1}}{2\left(\tau-\tau_{2}^{+}\right)\left(\tau-\tau_{2}^{-}\right)}, \\
& \phi_{1-}^{2}=\left(\frac{1}{d_{1-}}\right) \frac{A_{4} \tau^{2}+B_{4} \tau+C_{4}}{2\left(\tau-\tau_{2}^{+}\right)\left(\tau-\tau_{2}^{-}\right)}+\left(\frac{1}{d_{2-}}\right) \frac{A_{3} \tau^{2}+B_{3} \tau+C_{3}}{2\left(\tau-\tau_{2}^{+}\right)\left(\tau-\tau_{2}^{-}\right)}, \\
& \phi_{2-}^{1}=-\left(\frac{1}{d_{1-}}\right) \frac{A_{2} \tau^{2}+B_{2} \tau+C_{2}}{2\left(\tau-\tau_{1}^{+}\right)\left(\tau-\tau_{1}^{-}\right)}+\left(\frac{1}{d_{2-}}\right) \frac{A_{1} \tau^{2}+B_{1} \tau+C_{1}}{2\left(\tau-\tau_{1}^{+}\right)\left(\tau-\tau_{1}^{-}\right)}, \\
& \phi_{2-}^{2}=-\left(\frac{1}{d_{1-}}\right) \frac{A_{4} \tau^{2}+B_{4} \tau+C_{4}}{2\left(\tau-\tau_{1}^{+}\right)\left(\tau-\tau_{1}^{-}\right)}+\left(\frac{1}{d_{2-}}\right) \frac{A_{3} \tau^{2}+B_{3} \tau+C_{3}}{2\left(\tau-\tau_{1}^{+}\right)\left(\tau-\tau_{1}^{-}\right)} .
\end{aligned}
$$

The 12 constants $A_{j}, B_{j}, C_{j}, j=1,2,3,4$ are determined by imposing the conditions described below (3.57). Imposing the normalization condition $M_{+}(\tau=0)=\mathbb{I}$ yields

$$
C_{1}=-d_{1+}(\tau=0), \quad C_{2}=\frac{1}{C_{1}}, \quad C_{3}=-d_{1+}(\tau=0), \quad C_{4}=-\frac{1}{C_{3}} .
$$

Imposing that the $\phi_{i \pm}^{j}$ in (3.74) have the appropriate analyticity requirements at $\tau_{1,2}^{ \pm}$determines the coefficients $A_{j}, B_{j}$. We find that the $A_{j}$ and $B_{j}$ take the form $A_{j}=\tilde{A}_{j} / K, B_{j}=$ $\tilde{B}_{j} / K(j=1,2,3,4)$, where

$$
\begin{aligned}
K=\tau_{1}^{+} \tau_{2}^{+}\{ & 2 C_{1}^{2} D_{1+} D_{2+}\left(1+\left(\tau_{1}^{+}\right)^{2}\right)\left(1+\left(\tau_{2}^{+}\right)^{2}\right) \\
+ & C_{1}^{2}\left(-C_{1}^{2}\left(\tau_{1}^{+}-\tau_{2}^{+}\right)^{2}+D_{2+}^{2}\left(1+\tau_{1}^{+} \tau_{2}^{+}\right)^{2}\right) \\
+ & \left.D_{1+}^{2}\left(-D_{2+}^{2}\left(\tau_{1}^{+}-\tau_{2}^{+}\right)^{2}+C_{1}^{2}\left(1+\tau_{1}^{+} \tau_{2}^{+}\right)^{2}\right)\right\},
\end{aligned}
$$

and similar expressions for the $\tilde{A}_{j}$ and for the $\tilde{B}_{j}$. Here, we introduced the notation $D_{1+}=d_{1+}^{2}\left(\tau=\tau_{1}^{+}\right), \quad D_{2+}=d_{1+}^{2}\left(\tau=\tau_{2}^{+}\right), \quad D_{1-}=d_{1-}^{2}\left(\tau=\tau_{1}^{-}\right), \quad D_{2-}=d_{1-}^{2}\left(\tau=\tau_{2}^{-}\right)$, 
for convenience. Thus, as long as $K$ does not vanish, we have obtained a canonical factorization.

We recall that $A_{3}$ and $A_{4}$ have to satisfy the relations (3.61) and (3.63). We proceed to verify that $A_{1}, A_{2}, A_{3}, A_{4}$ indeed satisfy these relations. In doing so, we use the relations

$$
\frac{d_{1+}\left(\tau_{1}^{+}\right)}{d_{1-}\left(\tau_{1}^{-}\right)}=d_{1+}(\tau=0)=\frac{d_{1+}\left(\tau_{2}^{+}\right)}{d_{1-}\left(\tau_{2}^{-}\right)} .
$$

Next, we turn to $M(\rho, v)$. Using $d_{1-}(\infty)=d_{2-}(\infty)=1$ we obtain from (3.60),

$$
M(\rho, v)=\frac{1}{2}\left(\begin{array}{cc}
A_{1}+A_{2} & A_{1}-A_{2} \\
A_{1}-A_{2} & 4 \frac{\left(1+\frac{1}{4}\left(A_{1}-A_{2}\right)^{2}\right)}{\left(A_{1}+A_{2}\right)}
\end{array}\right) .
$$

The associated space-time solution is described by a stationary line element of the form

$$
d s_{4}^{2}=-\Delta(d t+\sigma)^{2}+\Delta^{-1}\left(e^{\psi}\left(d \rho^{2}+d v^{2}\right)+\rho^{2} d \phi^{2}\right),
$$

where both $\Delta$ and the one-form $\sigma=\sigma_{\phi} d \phi$ are determined in terms of the entries of $M(\rho, v)$. Namely, $\Delta$ is read off from (3.79) using (3.62), while the two-form $F=d \sigma$ is determined by

$$
\Delta^{2} * F=d B
$$

where $*$ denotes the Hodge dual in three dimensions with respect to the metric (2.1). Finally, $\psi$ is determined by (2.4).

The coefficients $A_{1}$ and $A_{2}$, and hence $\Delta$ and $B$, are very complicated functions of the Weyl coordinates $(\rho, v)$. To first order in the deformation parameter $\xi$ we obtain,

$$
\begin{aligned}
\Delta= & \frac{\tau_{2}^{+}}{\tau_{1}^{+}}+\mathcal{O}\left(\xi^{2}\right), \\
B= & 2 \xi \frac{\tau_{2}^{+}}{\rho\left(\tau_{0}^{+}-\tau_{0}^{-}\right) \tau_{1}^{+}\left(1+\tau_{1}^{+} \tau_{2}^{+}\right)\left(\tau_{1}^{+}-\tau_{0}^{-}\right)\left(\tau_{2}^{+}-\tau_{0}^{-}\right)} \\
& \times\left(\tau_{2}^{+} \tau_{0}^{-}-\left(\tau_{0}^{-}\right)^{2}+\tau_{1}^{+}\left(\tau_{2}^{+}+\tau_{0}^{-}\right)\left(1+\tau_{2}^{+} \tau_{0}^{-}\right)-\left(\tau_{2}^{+}\right)^{2}\left(1+\left(\tau_{0}^{-}\right)^{2}\right)\right. \\
& \left.\quad-\left(\tau_{1}^{+}\right)^{2}\left(1+\left(\tau_{2}^{+}\right)^{2}-\left(\tau_{2}^{+}\right) \tau_{0}^{-}+\left(\tau_{0}^{-}\right)^{2}\right)\right)+\mathcal{O}\left(\xi^{2}\right),
\end{aligned}
$$

while $\psi$ is undeformed at first order in $\xi$. Since $B$ is non-vanishing at first order in $\xi$, it induces a non-vanishing two-form $F$ given by (3.81). We have verified that $F$ is closed, i.e. $d F=0$, so that locally, $F=d \sigma$, with $\sigma=\sigma_{\phi} d \phi$.

The above shows that the Riemann-Hilbert factorization method constitutes a feasible and explicit method for obtaining deformed solutions to the field equations of gravitational field theories that may be hard to obtain by direct means, i.e. by directly solving the non-linear field equations.

We conclude by verifying the validity of the substitution rule. To this end, we focus on the region $v>m>0$, and consider the limit $\rho \rightarrow 0^{+}$. We obtain

$$
\begin{aligned}
\tau_{1}^{+} & \rightarrow-\frac{1}{2} \frac{\rho}{v-m} \rightarrow 0, & \tau_{2}^{+} & \rightarrow-\frac{1}{2} \frac{\rho}{v+m} \rightarrow 0, \\
d_{1+}(0) & \rightarrow e^{\xi / v}, & d_{1+}\left(\tau_{1}^{+}\right) & \rightarrow d_{1+}(0),
\end{aligned}
$$


Using this, we find in the limit $\rho \rightarrow 0^{+}$,

$$
\begin{aligned}
\tilde{A}_{1} & =-2 C_{1}^{7} \tau_{2}^{+}\left(\tau_{1}^{+}+\tau_{2}^{+}\right)+2 C_{1}^{5} \tau_{2}^{+}\left(\tau_{1}^{+}-\tau_{2}^{+}\right), \\
\tilde{A}_{2} & =2 C_{1}^{7} \tau_{2}^{+}\left(\tau_{1}^{+}-\tau_{2}^{+}\right)-2 C_{1}^{5} \tau_{2}^{+}\left(\tau_{1}^{+}+\tau_{2}^{+}\right), \\
K & \rightarrow 4 C_{1}^{6} \tau_{1}^{+} \tau_{2}^{+}
\end{aligned}
$$

and hence

$$
\begin{aligned}
& \frac{1}{2}\left(A_{1}+A_{2}\right) \rightarrow\left(\frac{v-m}{v+m}\right) \cosh \frac{\xi}{v}, \\
& \frac{1}{2}\left(A_{1}-A_{2}\right) \rightarrow \sinh \frac{\xi}{v} .
\end{aligned}
$$

Thus we infer

$$
\lim _{\rho \rightarrow 0^{+}} M(\rho, v)=\mathcal{M}(v),
$$

which verifies the validity of the substitution rule in the region $v>m>0$.

\section{Acknowledgments}

We would like to thank Cristina Câmara for valuable suggestions and discussions, and Thomas Mohaupt and Suresh Nampuri for valuable comments. J.C.S. gratefully acknowledges the support of the Gulbenkian Foundation through the scholarship program Novos Talentos em Matemática. This work was partially supported by FCT/Portugal through UID/MAT/04459/2013.

\section{A Explicit factorization}

We perform the explicit factorization of (3.21) by solving the associated vectorial factorization problem (3.22) column by column, and using (a generalized version of) Liouville's theorem (see the lemma on page 14 of [4]). We impose the normalization condition $\tilde{M}_{+}(\tau=0)=\mathbb{I}$.

To this end, we introduce the following notation: we denote by $\phi_{i \pm}^{j}$ the element in line $i$ and column $j$ of the matrix $\tilde{M}_{+}^{-1}$ or $\tilde{M}_{-}$in (3.21). We use

$$
\begin{aligned}
& H_{1}(\omega(\tau))=h_{1} \frac{\left(\tau-\tau_{\tilde{Q}}^{-}\right)\left(\tau-\tau_{\tilde{Q}}^{+}\right)}{\left(\tau-\tau_{0}^{-}\right)\left(\tau-\tau_{0}^{+}\right)}, \\
& H_{2}(\omega(\tau))=h_{2} \frac{\left(\tau-\tau_{\tilde{P}}^{-}\right)\left(\tau-\tau_{\tilde{P}}^{+}\right)}{\left(\tau-\tau_{0}^{-}\right)\left(\tau-\tau_{0}^{+}\right)} .
\end{aligned}
$$

The third line of (3.21) yields

$$
\begin{array}{ll}
\phi_{1+}^{1}=1, & \phi_{3-}^{1}=-1, \\
\phi_{1+}^{2}=0, & \phi_{3-}^{2}=0, \\
\phi_{1+}^{3}=0, & \phi_{3-}^{3}=0 .
\end{array}
$$


The second line of (3.21) yields the following. First we consider

$$
\phi_{2-}^{2}=-\frac{H_{1}}{H_{2}} \phi_{2+}^{2}=\frac{\alpha_{1} \tau+\beta_{1}}{\tau-\tau_{\tilde{P}}^{+}},
$$

by Liouville's theorem. This is solved by

$$
\begin{aligned}
\phi_{2+}^{2} & =\frac{\tau_{\tilde{Q}}^{-}}{\tau_{\tilde{P}}^{-}}\left(\frac{\tau-\tau_{\tilde{P}}^{-}}{\tau-\tau_{\tilde{Q}}^{-}}\right) \\
\phi_{2-}^{2} & =-\frac{h_{1}}{h_{2}} \frac{\tau_{\tilde{Q}}^{-}}{\tau_{\tilde{P}}^{-}}\left(\frac{\tau-\tau_{\tilde{Q}}^{+}}{\tau-\tau_{\tilde{P}}^{+}}\right) .
\end{aligned}
$$

Next, we consider

$$
\phi_{2-}^{1}=-\sqrt{2} H_{1} \phi_{1+}^{1}-\frac{H_{1}}{H_{2}} \phi_{2+}^{1}=\frac{\alpha_{2} \tau^{2}+\beta_{2} \tau+\gamma_{2}}{\left(\tau-\tau_{\tilde{P}^{+}}\right)\left(\tau-\tau_{0}^{+}\right)},
$$

by Liouville's theorem. This is solved by

$$
\begin{aligned}
\phi_{2+}^{1} & =-\sqrt{2} h_{2}\left(\frac{\tau-\tau_{\tilde{P}}^{-}}{\tau-\tau_{\tilde{Q}}^{-}}\right) \frac{\tau}{\tau-\tau_{0}^{-}} \frac{\left(\tau_{0}^{+}-\tau_{\tilde{Q}}^{+}\right)\left(\tau_{0}^{-}-\tau_{\tilde{P}}^{+}\right)}{\tau_{\tilde{Q}}^{+}\left(\tau_{0}^{+}-\tau_{0}^{-}\right)}, \\
\phi_{2-}^{1} & =-\frac{\sqrt{2} h_{1}}{\tau_{\tilde{Q}}^{+}}\left(\frac{\tau-\tau_{\tilde{Q}}^{+}}{\tau-\tau_{\tilde{P}}^{+}}\right) \frac{\left(\tilde{\alpha} \tau-\tau_{\tilde{P}}^{+} \tau_{0}^{+}\right)}{\left(\tau-\tau_{0}^{+}\right)},
\end{aligned}
$$

where

$$
\tilde{\alpha}=\frac{\tau_{\tilde{P}}^{+}\left(\tau_{0}^{+}-\tau_{\tilde{Q}}^{+}\right)+\tau_{\tilde{Q}}^{+} \tau_{0}^{+}+1}{\tau_{0}^{+}-\tau_{0}^{-}}
$$

In addition, we find

$$
\phi_{2+}^{3}=0, \quad \phi_{2-}^{3}=0 .
$$

Next, we turn to the first line of (3.21). We find

$$
\phi_{3+}^{3}=1, \quad \phi_{1-}^{3}=-1 .
$$

We also get

$$
\sqrt{2} H_{1} \phi_{2+}^{2}-\phi_{3+}^{2}=\phi_{1-}^{2}=\frac{\alpha_{3} \tau+\beta_{3}}{\tau-\tau_{0}^{+}},
$$

by Liouville's theorem. This is solved by

$$
\begin{aligned}
\phi_{3+}^{2} & =\sqrt{2} h_{1} \frac{\left(\tau_{\tilde{Q}}^{-}\left(\tau_{\tilde{P}}^{-}-\tau_{0}^{-}\right)-\tau_{0}^{+} \tau_{\tilde{P}}^{-}-1\right)}{\tau_{\tilde{P}}^{-}\left(\tau_{0}^{+}-\tau_{0}^{-}\right)} \frac{\tau}{\left(\tau-\tau_{0}^{-}\right)}, \\
\phi_{1-}^{2} & =\sqrt{2} h_{1} \frac{\left[\tau\left(\tau_{0}^{+}\left(\tau_{\tilde{P}}^{-}+\tau_{\tilde{Q}}^{-}\right)-\tau_{\tilde{Q}}^{-} \tau_{\tilde{P}}^{-}+1\right)\right]-\tau_{0}^{+} \tau_{\tilde{P}}^{-}\left(\tau_{0}^{+}-\tau_{0}^{-}\right)}{\tau_{\tilde{P}}^{-}\left(\tau_{0}^{+}-\tau_{0}^{-}\right)\left(\tau-\tau_{0}^{+}\right)} .
\end{aligned}
$$


Finally, we also obtain

$$
H_{1} H_{2} \phi_{1+}^{1}+\sqrt{2} H_{1} \phi_{2+}^{1}-\phi_{3+}^{1}=\phi_{1-}^{1}=\frac{\alpha_{4} \tau^{2}+\beta_{4} \tau+\gamma_{4}}{\left(\tau-\tau_{0}^{+}\right)^{2}}
$$

by Liouville's theorem. This yields

$$
\phi_{1-}^{1}=\frac{\alpha_{4} \tau^{2}+\beta_{4} \tau+\gamma_{4}}{\left(\tau-\tau_{0}^{+}\right)^{2}}
$$

as well as

$$
\begin{aligned}
\phi_{3+}^{1}=\frac{1}{\left(\tau-\tau_{0}^{+}\right)^{2}\left(\tau-\tau_{0}^{-}\right)^{2}}[ & -\left(\tau-\tau_{0}^{-}\right)^{2}\left(\alpha_{4} \tau^{2}+\beta_{4} \tau+\gamma_{4}\right) \\
& +h_{1} h_{2}\left(\tau-\tau_{\tilde{Q}}^{-}\right)\left(\tau-\tau_{\tilde{P}}^{-}\right)\left(\tau-\tau_{\tilde{Q}}^{+}\right)\left(\tau-\tau_{\tilde{P}}^{+}\right) \\
& \left.-2 h_{1} h_{2} \frac{\left(\tau_{0}^{+}-\tau_{\tilde{Q}}^{+}\right)\left(\tau_{0}^{-}-\tau_{\tilde{P}}^{+}\right)}{\left(\tau_{0}^{+}-\tau_{0}^{-}\right) \tau_{\tilde{Q}}^{+}} \tau\left(\tau-\tau_{0}^{+}\right)\left(\tau-\tau_{\tilde{Q}}^{+}\right)\left(\tau-\tau_{\tilde{P}}^{-}\right)\right]
\end{aligned}
$$

where the numerator of $\phi_{3+}^{1}$ has to have a double zero at $\tau=\tau_{0}^{+}$, which results in

$$
\begin{aligned}
\gamma_{4}= & h_{1} h_{2}\left(\tau_{0}^{+}\right)^{2} \\
\beta_{4} \tau_{0}^{+}= & -\alpha_{4}\left(\tau_{0}^{+}\right)^{2}-\gamma_{4}+\frac{h_{1} h_{2}}{\left(\tau_{0}^{+}-\tau_{0}^{-}\right)^{2}}\left(\tau_{0}^{+}-\tau_{\tilde{Q}}^{-}\right)\left(\tau_{0}^{+}-\tau_{\tilde{P}}^{-}\right)\left(\tau_{0}^{+}-\tau_{\tilde{Q}}^{+}\right)\left(\tau_{0}^{+}-\tau_{\tilde{P}}^{+}\right), \\
\alpha_{4}= & h_{1} h_{2}\left(1-\frac{2 \tilde{Q}}{h_{1} \rho\left(\tau_{0}^{+}-\tau_{0}^{-}\right)}\right)\left(1-\frac{2 \tilde{P}}{h_{2} \rho\left(\tau_{0}^{+}-\tau_{0}^{-}\right)}\right) \\
& -2 h_{1} h_{2} \frac{\left(\tau_{\tilde{Q}}^{+}-\tau_{\tilde{P}}^{+}\right)\left(\tau_{0}^{+}-\tau_{\tilde{P}}^{-}\right)\left(\tau_{0}^{+}-\tau_{\tilde{Q}}^{+}\right)}{\tau_{\tilde{Q}}^{+}\left(\tau_{0}^{+}-\tau_{0}^{-}\right)^{2}}
\end{aligned}
$$

Having obtained the factorization of (3.21), we extract the matrix $M(\rho, v)$ given in (3.23). In obtaining the expressions given in (3.24), we used the following relation,

$$
\frac{2 \tilde{Q}}{\rho}=\frac{\left(\tau_{0}^{+}-\tau_{\tilde{Q}}^{+}\right)\left(\tau_{0}^{-}-\tau_{\tilde{Q}}^{+}\right)}{\tau_{\tilde{Q}}^{+}}=-\frac{\left(\tau_{0}^{+}-\tau_{\tilde{Q}}^{-}\right)\left(\tau_{0}^{+}-\tau_{\tilde{Q}}^{+}\right)}{\tau_{0}^{+}}=\tau_{\tilde{Q}}^{+}+\tau_{\tilde{Q}}^{-}-\tau_{0}^{+}-\tau_{0}^{-} .
$$

Open Access. This article is distributed under the terms of the Creative Commons Attribution License (CC-BY 4.0), which permits any use, distribution and reproduction in any medium, provided the original author(s) and source are credited.

\section{References}

[1] P. Breitenlohner and D. Maison, On the Geroch group, Ann. Inst. H. Poincaré Phys. Theor. 46 (1987) 215 [INSPIRE].

[2] J.H. Schwarz, Classical symmetries of some two-dimensional models coupled to gravity, Nucl. Phys. B 454 (1995) 427 [hep-th/9506076] [INSPIRE].

[3] H. Lü, M.J. Perry and C.N. Pope, Infinite-dimensional symmetries of two-dimensional coset models coupled to gravity, Nucl. Phys. B 806 (2009) 656 [arXiv:0712.0615] [InSPIRE]. 
[4] M.C. Câmara, G.L. Cardoso, T. Mohaupt and S. Nampuri, A Riemann-Hilbert approach to rotating attractors, JHEP 06 (2017) 123 [arXiv:1703.10366] [INSPIRE].

[5] S. Mikhlin and S. Prössdorf, Singular integral operators, Springer-Verlag, Berlin Germany, (1986).

[6] M.C. Câmara, Toeplitz operators and Wiener-Hopf factorisation: an introduction, Concrete Operators 4 (2017) 130 [arXiv:1710.11572].

[7] D. Maison, Geroch group and inverse scattering method, in Conference on nonlinear evolution equations: integrability and spectral methods, Como Italy, 4-15 July 1988 [INSPIRE].

[8] H. Nicolai, Two-dimensional gravities and supergravities as integrable system, Lect. Notes Phys. 396 (1991) 231 [INSPIRE].

[9] D. Katsimpouri, A. Kleinschmidt and A. Virmani, Inverse scattering and the Geroch group, JHEP 02 (2013) 011 [arXiv:1211.3044] [INSPIRE].

[10] D. Katsimpouri, A. Kleinschmidt and A. Virmani, An inverse scattering formalism for STU supergravity, JHEP 03 (2014) 101 [arXiv:1311.7018] [INSPIRE].

[11] D. Katsimpouri, A. Kleinschmidt and A. Virmani, An inverse scattering construction of the JMaRT fuzzball, JHEP 12 (2014) 070 [arXiv:1409.6471] [INSPIRE].

[12] B. Chakrabarty and A. Virmani, Geroch group description of black holes, JHEP 11 (2014) 068 [arXiv: 1408.0875] [INSPIRE].

[13] S. Ferrara and R. Kallosh, Supersymmetry and attractors, Phys. Rev. D 54 (1996) 1514 [hep-th/9602136] [INSPIRE].

[14] S. Ferrara and R. Kallosh, Universality of supersymmetric attractors, Phys. Rev. D 54 (1996) 1525 [hep-th/9603090] [INSPIRE].

[15] P. Figueras, E. Jamsin, J.V. Rocha and A. Virmani, Integrability of five dimensional minimal supergravity and charged rotating black holes, Class. Quant. Grav. 27 (2010) 135011 [arXiv: 0912.3199] [INSPIRE].

[16] L.V. Ahlfors, Complex analysis, third edition, McGraw-Hill International Book Company, U.S.A., (1984).

[17] D. Rasheed, The rotating dyonic black holes of Kaluza-Klein theory, Nucl. Phys. B 454 (1995) 379 [hep-th/9505038] [INSPIRE].

[18] T. Matos and C. Mora, Stationary dilatons with arbitrary electromagnetic field, Class. Quant. Grav. 14 (1997) 2331 [hep-th/9610013] [INSPIRE].

[19] F. Larsen, Rotating Kaluza-Klein black holes, Nucl. Phys. B 575 (2000) 211 [hep-th/9909102] [INSPIRE].

[20] D. Astefanesei, K. Goldstein, R.P. Jena, A. Sen and S.P. Trivedi, Rotating attractors, JHEP 10 (2006) 058 [hep-th/0606244] [INSPIRE].

[21] R. Emparan and A. Maccarrone, Statistical description of rotating Kaluza-Klein black holes, Phys. Rev. D 75 (2007) 084006 [hep-th/0701150] [INSPIRE].

[22] D.D.K. Chow and G. Compère, Black holes in $N=8$ supergravity from $\mathrm{SO}(4,4)$ hidden symmetries, Phys. Rev. D 90 (2014) 025029 [arXiv:1404.2602] [INSPIRE].

[23] E. Bergshoeff, R. Kallosh and T. Ortín, Stationary axion/dilaton solutions and supersymmetry, Nucl. Phys. B 478 (1996) 156 [hep-th/9605059] [INSPIRE].

[24] K. Behrndt, D. Lüst and W.A. Sabra, Stationary solutions of $N=2$ supergravity, Nucl. Phys. B 510 (1998) 264 [hep-th/9705169] [INSPIRE].

[25] G.L. Cardoso, A. Ceresole, G. Dall'Agata, J.M. Oberreuter and J. Perz, First-order flow equations for extremal black holes in very special geometry, JHEP 10 (2007) 063 [arXiv:0706.3373] [INSPIRE]. 